bitte auf den kommenden ungeraden Seiten und

Guillaume Cheikbossian and Philippe Mahenc

auf den geraden Seiten als Seitentitel setzen.

\title{
On the Difficulty of Collusion in the Presence of a More Efficient Outsider
}

\author{
by \\ Guillaume Cheikbossian and Philippe Mahenc*
}

Received May 9, 2016; in revised form July 12, 2017; accepted August 16, 2017

\begin{abstract}
We study the ability of several identical firms to collude in the presence of a more efficient firm, which does not take part in their collusive agreement. The cartel firms adopt stick-and-carrot strategies, while the efficient firm plays its one-period best-response function, regardless of the history of play. We characterize the most collusive symmetric punishment, which maximizes the scope for collusion. We then find that either a lower cost disadvantage or a smaller cartel size facilitates collusion. Finally, we compare our results with those obtained in the standard setup where all firms participate in the collusive agreement.
\end{abstract}

Keywords: repeated game, tacit collusion, optimal punishments, cost asymmetry, outsider

JEL classification code: C73, D43, L13

\section{Introduction}

Entry presents a serious challenge to a cartelized industry, especially when the entrant is not taking part in the cartel agreement. Levenstein and Suslow (2006) compile several case studies and conclude that in many instances new entry is not necessarily followed by an invitation to join the cartel, but rather results in what they call a "disruptive cartel." In other words, cartel firms respond to entry with punitive or predatory behavior, which in turn can jeopardize the future of the cartel. In a sample of 54 international cartel breakdowns in 22 industries, Griffin (1989) indeed finds that new entry into the market is just as important as cheating and defection for explaining market breakdown - each cause representing one-third of cartel failures. ${ }^{1}$

* Guillaume Cheikbossian (corresponding author) and Philippe Mahenc: Université de Montpellier (LAMETA) \& TSE, Montpellier, France. We thank seminar participants in Montpellier and two anonymous referees for helpful comments and suggestions. This research received funding from the French Agence nationale de la Recherche as part of the project GREENGO - New Tools for Environmental Governance: the role of NGOs - ANR-15-CE050008. We thank Patrick González for helping us with the figures.

${ }^{1}$ See Levenstein and Suslow (2006) for a survey of other empirical and case studies of cartel breakdowns. 
In the present analysis, we take as given the presence of an outsider in a cartelized industry and we analyze whether tacit collusion between cartel members can yet be maintained through the use of a punishment strategy. ${ }^{2}$ Furthermore, echoing the "disruptive cartel" situation mentioned by Levenstein and Suslow (2006), we also consider that the incumbent cartel always responds noncooperatively to the outsider's strategy. Finally, the outsider is assumed to be more efficient than the cartel firms. A general motivation for the assumption of a cost-advantaged outsider is that the lack of competition implied by a cartel agreement may result in $X$-inefficient behaviors à la Leibenstein (1966). Furthermore, as argued by Baker (1995), cartel firms are less likely to adopt new innovations. And the persistence of productive inefficiency within the cartel could be the main reason for which the entry of a more efficient outsider occurred in the first place.

In this paper, we thus employ an oligopoly model in which $n$ symmetric firms, called the cartel, plus one cost-advantaged firm, called the outsider, play a Cournot game over an infinite horizon. The firms of the cartel adopt two-phase stick-and-carrot punishment schemes à la Abreu $(1986,1988)$ to support their joint-profit-maximizing behavior. In other words, following any deviation, the firms of the cartel conform to a stick, or punishment, phase in which they produce a very high quantity (the punishment output level) for one period (thus resulting in very low market price and profits during that period) to generate a carrot in the possibility of a subsequent return to collusive behavior. Deviations from the punishment simply cause it to begin again. As for the outsider, it is assumed to play in every period its best response to the other firms' strategies regardless of the history. We then focus on a subgame-perfect equilibrium that supports perfect collusion within the cartel and noncooperation between the cartel and the outsider. In this equilibrium, collusion among the firms of the cartel makes them act as if they were a single firm, and hence the outcome corresponds to that of a Cournot duopoly game.

There typically exists an infinity of punishment output levels that support perfect collusion within the cartel as a subgame-perfect outcome. We focus on the punishment output that supports collusion for the largest range of discount factors and thus makes it as easy as possible for cartel firms to collude. This singles out what we call the most collusive symmetric punishment (MCSP) in that it most relaxes the incentivecompatibility constraints, in both the collusive and the punishment phase. ${ }^{3}$ As we will see, this requires equalizing the net gains of cheating in the two phases. Also, the MCSP crucially depends on both the size of the cartel and the cost asymmetry between the cartel firms and the outsider. More specifically, we show that perfect collusion within the

\footnotetext{
${ }^{2}$ In the literature, the term "cartel" refers to both explicit collusion and implicit collusion (see Levenstein and Suslow, 2006). In the present analysis, we consider the case of implicit collusion, which is more difficult to detect and prove than explicit collusion.

${ }^{3}$ We prefer to introduce a new term to describe the punishment scheme maximizing the scope for collusion among participating firms rather than to refer to Abreu's "optimal penal code." This is because Abreu $(1986,1988)$ derives optimal punishments within the class of symmetrical punishments, i.e., punishments that assume that all firms act identically. This is not the case in our analysis, since one firm does not participate in the collusive agreement.
} 
cartel requires harsher punishment output levels when the cost asymmetry is sufficiently small for a given number of cartel firms. However, the MCSP is as likely to be harsher as milder with a larger number of cartel firms.

Next, we characterize the minimum value for the discount factor above which perfect collusion is sustainable as a subgame-perfect outcome. This provides us with a measure of the ease of collusion between the cartel firms. It turns out that a reduction in the cartel size, as well as a lower cost disadvantage, facilitates collusion. However, once the cartel gets large, the cost disadvantage has no effect on the difficulty of collusion.

Finally, we compare our results with those obtained by Motta (2004) in a similar model with linear demand and cost functions but with all firms being identical and participating in the collusive agreement with the use of stick-and-carrot strategies. Actually, collusion between $n$ firms against a more efficient outsider is easier to sustain than collusion between $n+1$ identical firms with no outsider. Intuitively, having an outsider to the cartel allows stronger equilibrium punishments, which then facilitate collusion, than in the case where all firms are colluding.

This paper is related to the literature on tacit collusion in oligopolistic markets. Since the work of Friedman (1971), the vast majority of analysis has assumed identical firms and that all of them participate in the cartel agreement. Some recent works have analyzed the sustainability of tacit collusion among heterogeneous firms. These works emphasize that, in general, asymmetries in the cost functions hinder collusion, with the results depending crucially upon the profit-sharing rule (see, e.g., Rothschild, 1999; Vasconcelos, 2005) and on whether side payments between firms are allowed (see, e.g., Miklós-Thal, 2011). Yet, most analyses continue to assume that all the firms of the industry participate in the collusive agreement. There is, however, a pair of recent articles that analyze the sustainability of tacit collusion in the presence of outsider(s). In a quantity-setting supergame, Escrihuela-Villar (2008a,b) investigates the incentives to merge when the collusive agreement is not all-inclusive. Considering both Nashreversion and stick-and-carrot strategies, these works establish a link with the literature on cartel stability with a Cournot fringe in a static setting (see Shaffer, 1995). However, all firms are assumed to be identical. More related to the present work is the paper by Bos and Harrington (2010). They first provide many examples where larger firms do not participate in the cartel agreement, which is consistent with the assumption of a cost-advantaged outsider. ${ }^{4}$ In turn, they develop a model to endogenize the composition of a cartel with firms that are heterogeneous with respect to capacities. However, they use a price-setting supergame and assume that the collusive outcome is supported by the threat of infinite reversion to the static Nash equilibrium with zero profit.

${ }^{4}$ For instance, the vitamin B1 cartel's global market share fell from $70 \%$ down to $52 \%$ during the first 3 years of collusion because the Chinese suppliers were absent from the cartel. Another example is the vitamin B2 cartel, from which the U.S. producer Coors was notably absent. Bos and Harrington (2010) also point out that the 13-year-long European industrial tubes cartel excluded at least two significant producers. Finally, in the Danish district heating pipes cartel, the Swedish firm Powerpipe, which was a sizable competitor, preferred not to join the cartel. 
Our analysis can be viewed as complementary to all these papers in that we analyze the ability of several firms to sustain collusion in quantities through the use of stickand-carrot strategies when they face a more efficient (and nonparticipating) firm.

The rest of this paper is organized as follows. In section 2, we describe the basic model. In section 3, we explore the infinitely repeated game and provide necessary and sufficient conditions for perfect collusion within the cartel to be sustained as a subgame-perfect outcome through the use of stick-and-carrot strategies à la Abreu (1986, 1988). Section 4 derives the MCSP, depending on the size of the cartel and on the cost asymmetry between the cartel firms and the outsider. In the section after, we characterize the resulting minimum discount factors for sustaining perfect collusion and investigate how the ease of collusion is affected by the size of the cartel or the cost asymmetry. In that section, we also compare our results with those obtained in the standard setup where all firms participate in the collusive agreement. Finally, section 6 offers some concluding comments.

\section{The Model}

\subsection{The Stage Game}

We start by specifying the details of the stage game $G$. There are $n+1$ firms, indexed $i=$ $0,1,2, \ldots, n$, which produce a homogenous product. All firms of the cartel, $i=1,2, \ldots, n$, incur the same marginal cost $c \geq 0$, whereas the outsider $i=0$ has a marginal cost equal to 0. Again, the discrepancy in cost between the cartel firms and the outsider is consistent with Leibenstein's (1966) view that the lack of competitive pressure involves some degree of $X$-inefficiency. Indeed, the cartel agreement leads to a situation where competitive pressure is light and incentives to innovate are weak, as argued by Baker (1995). In this context, by contrast, the outsider has strong incentives to innovate that make it more efficient than cartel firms. In this view, the parameter $c$ measures the degree of $X$-inefficiency of the cartel relative to the outsider. Another interpretation of the cost discrepancy is that the outsider benefits from subsidies or tax exemption from its government while the $n$ other firms are not subsidized due to a more stringent regulation or the lack of support of the industry by their own governments. In this respect, Gilbert (1996) points out that the robustness of collusion between small producers in world markets for primary commodities such as cocoa, coffee, natural rubber, and cotton is a major issue. ${ }^{5}$

Let $q_{i} \in \mathbb{R}_{+}$be the output of firm $i$, for $i=0,1, \ldots, n$. Then $Q=\sum_{i=0}^{n} q_{i}$ is the aggregate output. The inverse demand function is given by $p(Q)=\max \{0,1-Q\}$.

\footnotetext{
${ }^{5}$ Indeed, a typical example is that of subsidies to cotton farmers, which are more developed in some parts of the world than in others. Thus, such subsidies are common practices on the part of governments of the United States, the European Union, China, or India, despite the World Trade Organisation's ruling these practices illegal: http://www.guardian.co.uk/globaldevelopment/poverty-matters/2011/may/24/american-cotton-subsidies-illegal-obama-must-ac t, http://www.guardian.co.uk/environment/2010/nov/15/cotton-subsidies-west-africa, http:/ /www.washingtonpost.com/wp-dyn/content/article/2010/06/02/AR2010060204228.html.
} 
Thus, the payoff function of firm 0 is $\pi_{0}\left(q_{0}, q_{1}, \ldots, q_{n}\right)=p(Q) q_{0}$, while that of firm $i$, for $i=1, \ldots, n$, is $\pi_{i}\left(q_{0}, q_{1}, \ldots, q_{n}\right)=[p(Q)-c] q_{i}$.

Let $r_{i}\left(q_{-i}\right)$, for $i=0, \ldots, n$, be the firm $i$ 's single-period best-response function to the vector of output levels $q_{-i}=\left(q_{0}, \ldots, q_{i-1}, q_{i+1} \ldots, q_{n}\right)$, so that $r_{i}\left(q_{-i}\right)$ satisfies $\pi_{i}\left(r_{i}\left(q_{-i}\right), q_{-i}\right) \geq \pi_{i}\left(q_{i}, q_{-i}\right)$ for all $\left(q_{i}, q_{-i}\right) \in \mathbb{R}_{+}^{n+1}$. Thus, we have

$$
r_{0}\left(q_{-0}\right)=\max \left\{0, \frac{1-\sum_{i=1}^{n} q_{i}}{2}\right\}, \quad \text { and } \quad r_{i}\left(q_{-i}\right)=\max \left\{0, \frac{1-q_{0}-\sum_{j \neq i} q_{j}-c}{2}\right\}
$$

for $i=1, \ldots, n$.

We focus on symmetric equilibria in the sense that all firms of the cartel produce the same level of output. For the sake of simplicity, we will write $r_{0}\left(q_{-0}\right)=r_{0}(x)$ if $q_{-0}=(x, x, x, \ldots, x) \in \mathbb{R}_{+}^{n}$ and $r_{i}\left(q_{0}, q_{-i}\right)=r_{i}\left(q_{0}, x\right)$ if $\left(q_{0}, q_{-i}\right)=\left(q_{0}, x, x, x, \ldots, x\right) \in$ $\mathbb{R}_{+}^{n+1}$. The noncooperative equilibrium, both within the cartel and between the cartel and the outsider, is characterized by a pair of individual output levels $\left(q_{0}^{N}, q^{N}\right)$ such that $r_{0}\left(q^{N}\right)=q_{0}^{N}$ and $r_{i}\left(q_{0}^{N}, q^{N}\right)=q^{N}$ for $i=1, \ldots, n$. We obtain

$$
\left(q_{0}^{N}, q^{N}\right)=\left(\frac{1+n c}{n+2}, \frac{1-2 c}{n+2}\right)
$$

and so the market clears at price $p^{N}=(1+n c) /(n+2)$.

To guarantee that each firm in the stage game has a positive market share, we make the following assumption.

Assumption $1 \quad c<1 / 2$.

Let $\pi_{0}^{N} \equiv \pi_{0}\left(q_{0}^{N}, q^{N}, \ldots, q^{N}\right)$, the payoff of the outsider, and $\pi^{N} \equiv \pi_{i}\left(q_{0}^{N}, q^{N}, \ldots, q^{N}\right)$, for $i=1, \ldots, n$, the identical payoff for each less-efficient firm when there is noncollusion. We have

$$
\pi^{N}=\left[\frac{1-2 c}{n+2}\right]^{2} \text { and } \pi_{0}^{N}=\left[\frac{1+n c}{n+2}\right]^{2}
$$

with $\pi_{0}^{N}>\pi^{N}$.

\subsection{Collusion}

Assume now that the firms of the cartel collude and jointly choose a common level of individual output $q$ so as to maximize the sum of their profits. Yet, they continue playing noncooperatively with the outsider, so that the outcome resembles that of a Cournot duopoly game between the cartel and the outsider. The collusive output from the viewpoint of the cartel is given by the maximization of $\sum_{i=1}^{n} \pi_{i}\left(q_{0}, q, \ldots, q\right)=(p(Q)-$ c) $n q$ with respect to $q$, where $Q=n q+q_{0}$. Under collusion, cartel firms do not respond to each other, because they do not take each other's production level as given. Yet, the common best response of the cartel firms to the outsider is again defined on the firm level. Thus, the best response of any collusive firm of the cartel to the output level $q_{0}$ produced by the outsider is given by the function $R_{i}\left(q_{0}\right)=\max \left\{0,\left(1-q_{0}-c\right) / 2 n\right\}$, for $i=1, \ldots, n$. 
And the noncooperative equilibrium between the cartel firms - which act as if they were a single firm - and the outsider is thus given by a pair of individual output levels $\left(q_{0}^{C}, q^{C}\right)$ such that $r_{0}\left(q^{C}\right)=q_{0}^{C}$ and $R_{i}\left(q_{0}^{C}\right)=q^{C}$, for $i=1, \ldots, n$. Hence, when the cartel firms fully collude with each other, we obtain that $\left.\left(q_{0}^{C}, q^{C}\right)=((1+c) / 3,(1-2 c) / 3 n)\right)$.

Let $\pi_{0}^{C} \equiv \pi_{0}\left(q_{0}^{C}, q^{C}, \ldots, q^{C}\right)$, be the payoff of the outsider, and $\pi^{C} \equiv \pi_{i}\left(q_{0}^{C}, q^{C}, \ldots, q^{C}\right)$ be, for $i=1, \ldots, n$, the identical payoff for each less-efficient firm when there is collusion. The market price equilibrium is $p^{C}=(1+c) / 3$, and then

$$
\pi^{C}=\frac{1}{n}\left(\frac{1-2 c}{3}\right)^{2}
$$

and

$$
\pi_{0}^{C}=\left(\frac{1+c}{3}\right)^{2}
$$

We can verify that $\pi^{C}-\pi^{N}=n^{2}-5 n+4>0$ if and only if $n>4$. This is consistent with the so-called merger paradox identified by Salant, Switzer, and Reynolds (1983). They indeed show - in the linear demand model with constant marginal costs and homogenous products - that mergers are not beneficial to the participating firms unless more than $80 \%$ of them collude. Hence, to make the problem interesting, we will assume the following.

Assumption $2 n>4$.

Notice also that, under Assumptions 1 and $2, \pi_{0}^{C}-\pi_{0}^{N}=(1-2 c)(n-1)>0$. In other words, the outsider always benefits from collusion of the $n$ other firms. This is because collusive firms reduce their output levels, which in turn increases both the market share and the profit of the outsider.

\section{The Infinitely Repeated Game}

\subsection{Definition of the Game}

The $n+1$ firms play an infinitely repeated game with discounting. Time is discrete, and dates are denoted by $t=0,1,2, \ldots$. Let $G^{\infty}(\delta)$ be the repeated game obtained by repeating $G$ infinitely often, where $\delta \in(0,1)$ is the discount parameter per period for each player. We assume that the output produced by each firm in each period is perfectly observed by all firms. Let $q_{0}(t) \in \mathbb{R}_{+}$and $q_{-0}(t) \equiv\left(q_{1}(t), \ldots, q_{n}(t)\right) \in \mathbb{R}_{+}^{n}$ be respectively the output level produced by the outsider and the vector of outputs produced by the $n$ firms of the cartel in period $t$. Hence, a (finite) history in period $t \geq 1$ is denoted $h(t) \equiv$ $\left(h_{0}(t), h_{-0}(t)\right)$, where $h_{0}(t)=\left(q_{0}(0), \ldots, q_{0}(t-1)\right)$ and $h_{-0}(t)=\left(q_{-0}(0), \ldots, q_{-0}(t-1)\right)$. Let $H_{t}$ be the set of $t$-period histories. We further define the initial history to be the null set, $H_{0} \equiv\{\emptyset\}$, and $H$ to be the set of all possible publicly observable histories, $H \equiv \bigcup_{t=0}^{\infty} H_{t}$. A pure strategy for firm $i$ in $G^{\infty}(\delta)$, for $i=0,1,2, \ldots, n$, is an infinite sequence of functions $\sigma_{i}=\left\{\sigma_{i}^{t}\right\}_{t=1}^{\infty}$, where $\sigma_{i}^{t}: H_{t} \rightarrow \mathbb{R}_{+}$is a mapping from the set of all possible histories up to $t-1$ (included) into the set of output levels. 
Let $\sigma_{-0} \equiv\left(\sigma_{1}, \ldots, \sigma_{n}\right)$ be the strategy profile of the cartel firms. Any strategy profile $\sigma \equiv\left(\sigma_{0}, \sigma_{-0}\right)$ generates an output path $\left\{q_{0}(t), q_{-0}(t)\right\}_{t=0}^{\infty}$ defined by $\left(q_{0}(0), q_{-0}(0)\right)=$ $\sigma(\emptyset)$ and $\left(q_{0}(t), q_{-0}(t)\right)=\left(\sigma_{0}^{t}, \sigma_{1}^{t}, \ldots, \sigma_{n}^{t}\right)$ for all $t \geq 1$. An output path $\left\{q_{0}(t), q_{-0}(t)\right\}_{t=0}^{\infty}$ thus implies an infinite stream of stage-game payoffs $\left\{\pi_{i}\left(q_{0}(t), q_{-0}(t)\right)\right\}_{t=0}^{\infty}$ for firms $i=$ $0,1, \ldots, n$. The discounted payoff to firm $i$ from the infinite sequence of stage-game payoffs $\left\{\pi_{i}(t)\right\}_{t=0}^{\infty}$ is given by $\sum_{t=0}^{\infty} \delta^{t} \pi_{i}(t)$, so that its payoff in $G^{\infty}(\delta)$ obtained with the strategy profile $\sigma$ is

$$
\pi_{i}^{\delta}(\sigma)=\sum_{t=0}^{\infty} \delta^{t} \pi_{i}\left(q_{0}(\sigma)(t), q_{-0}(\sigma)(t)\right) .
$$

A strategy profile $\sigma$ is a Nash equilibrium in $G^{\infty}(\delta)$ if $\sigma_{0}$ is a best response to $\sigma_{-0}$ and if $\sigma_{i}$, for $i=1, \ldots, n$, is a best response to $\sigma_{-i}=\left(\sigma_{1}, \ldots, \sigma_{i-1}, \sigma_{i+1}, \ldots, \sigma_{n}\right)$ and to $\sigma_{0}$. And it is a subgame-perfect equilibrium in $G^{\infty}(\delta)$ if after every history, the continuation of $\sigma$ is a Nash equilibrium in the corresponding subgame. We will restrict attention to stationary subgame-perfect equilibria (SSPEs), i.e., equilibria in which after any history, a stationary profile of actions is played thereafter, and that also satisfy the additional requirement of symmetry within the cartel, in the sense that all firms of the cartel produce the same level of output at every history.

\subsection{The Two-Phase Punishment-Collusion Scheme}

The firms of the cartel adopt stick-and-carrot strategies à la Abreu (1986, 1988) to support the joint-profit maximizing level of output as a subgame-perfect equilibrium. Formally, for any level of output $q_{0}$ produced by the outsider in period $t$, consider two levels of output produced by any firm of the cartel as functions of $q_{0}$, i.e., $\left(\mathcal{S}\left(q_{0}\right), \mathcal{C}\left(q_{0}\right)\right)$, and define a two-phase punishment-collusion profile $\sigma\left(\mathcal{S}\left(q_{0}\right), \mathcal{C}\left(q_{0}\right)\right)$ to be stick-andcarrot strategies in which all firms of the cartel produce $\mathcal{S}\left(q_{0}\right)$ in one period and thereafter play $\mathcal{C}\left(q_{0}\right)$, with any deviation from these strategies causing this prescription to be repeated. Intuitively, $\mathcal{S}\left(q_{0}\right)$ is the stick, involving a high level of output, and $\mathcal{C}\left(q_{0}\right)$ is the carrot, involving a low and collusive level of output. The punishment specifies a singleperiod penalty followed by repeated play of the carrot. Deviations from the punishment simply cause it to begin again.

We are concerned with the best subgame-perfect equilibrium from the viewpoint of the cartel firms, and we further assume that the outsider plays in every period its best response to the other firms' strategies regardless of the history, i.e., $\sigma_{0}^{t}=r_{0}\left(q_{-0}(t)\right)$ for all $t$. During the collusive phase within the cartel, we thus pay attention to the cartelcollusive output $q^{C}=\mathcal{C}\left(q_{0}^{C}\right)=R_{i}\left(q_{0}^{C}\right)$ for $i=1, \ldots, n$ while the outsider's best response is $r_{0}\left(q^{C}\right)=q_{0}^{C}$. During the punishment phase, we are concerned with the level of output $x$ that will be determined endogenously, i.e., $\mathcal{S}\left(q_{0}^{x}\right)=x$ while the outsider's best response is $r_{0}(x)=q_{0}^{x}$. This strategy profile, denoted by $\sigma^{*}\left(\mathrm{x}, \mathrm{q}^{C}\right)$, where $\mathrm{x} \equiv\left(q_{0}^{x}, x, x, \ldots, x\right)$ and $\mathrm{q}^{C} \equiv\left(q_{0}^{C}, q^{C}, q^{C}, \ldots, q^{C}\right)$, specifies the following strategies for $i=1, \ldots, n$ : at $t=0$, 
$\sigma_{i}^{0}=q^{C}$, and, at $t \geq 1$,

$$
\sigma_{i}^{t}= \begin{cases}q^{C} & \text { if } \sigma_{j}^{\tau}=q^{C} \text { for } j=1, \ldots, n, \text { and all } \tau<t \\ q^{C} & \text { if } \sigma_{j}^{\tau}=x \text { for } j=1, \ldots, n, \text { and } \tau=t-1 \\ x & \text { otherwise }\end{cases}
$$

The profile $\sigma^{*}\left(\mathrm{x}, \mathrm{q}^{C}\right)$ can sustain collusion among the $n$ firms as a subgame-perfect equilibrium output path if and only if a single-period deviation from the strategy (and sticking to it subsequently) after any history is not profitable for any firm of the cartel. There are two kinds of histories to check. The first is that no single deviation has taken place in the previous period, so that the $n$ firms are in a collusive phase. The second state to check is the one where a firm deviated from the collusive agreement in the previous period, so that the $n$ firms are currently in the punishment phase.

Suppose first that the firms of the cartel are in a collusive phase, i.e., they all produce $q^{C}$ and the outsider produces $q_{0}^{C}$, and that firm $i$ considers deviating from $q^{C}$. The deviator maximizes $\pi_{i}\left(q_{0}^{C}, q^{C}, \ldots, q_{i}, \ldots, q^{C}\right)=\max \left\{0,\left(1-q_{i}-(n-1) q^{C}-q_{0}^{C}-c\right) q_{i}\right\}$ with respect to $q_{i}$, and hence the optimal deviation output is given by $q^{D}=(n+1)(1-2 c) / 6 n$.

Let $\pi^{D} \equiv \pi_{i}\left(q_{0}^{C}, q^{C}, \ldots, q^{D}, \ldots, q^{C}\right)$ be the optimal deviation profit for each firm $i$ of the cartel. We have

$$
\pi^{D}=\left[\frac{(n+1)(1-2 c)}{6 n}\right]^{2} .
$$

The temptation to deviate is thus given by

$$
\pi^{D}-\pi^{C}=\left[\frac{(n-1)(1-2 c)}{6 n}\right]^{2},
$$

which is decreasing in $c$ but increasing in $n$. Hence, the greater the cost disadvantage, the lower is the temptation to deviate from the collusive phase for each firm of the cartel. However, a larger size of the cartel increases the temptation to deviate.

Now, let $\pi^{P}(x) \equiv \pi_{i}\left(r_{0}(x), x, \ldots, x\right)$ denote the payoff of any firm of the cartel when they all produce $x$ units of output while the outsider simultaneously best responds to this level. Moreover, let $V^{P}$ be the present discounted value of the payoffs following a deviation, that is,

$$
V^{P}=\pi^{P}(x)+\frac{\delta}{1-\delta} \pi^{C} .
$$

No firm of the cartel has an incentive to deviate from $\sigma^{*}\left(\mathrm{x}, \mathrm{q}^{C}\right)$ in the collusive phase if and only if

$$
\pi^{D}+\delta V^{P} \leq \frac{1}{1-\delta} \pi^{C} .
$$

In other words, the one-shot deviation gain from the collusive phase plus the discounted payoff of entering the punishment phase next period must not exceed the payoff from 
continued collusion. Rearranging this inequality and using (4), we obtain the incentivecompatibility constraint along the collusive path:

$$
\delta \geq \delta^{C}(x) \equiv \frac{\pi^{D}-\pi^{C}}{\pi^{C}-\pi^{P}(x)} .
$$

Suppose now that one firm has deviated from $\sigma^{*}\left(\mathrm{x}, \mathrm{q}^{C}\right)$ in the previous period, so that the firms of the cartel are in the punishment phase in the current period. Suppose also that firm $i$ considers deviating from $x$ to get the optimal deviation payoff during the punishment phase, i.e., $\pi^{D P}(x) \equiv \pi_{i}\left(r_{0}(x), x, \ldots, q^{D P}(x), \ldots, x\right)$, where $q^{D P}(x) \equiv$ $\arg \max _{q_{i}} \pi_{i}\left(r_{0}(x), x, \ldots, q_{i}, \ldots, x\right)$. No firm of the cartel has an incentive to deviate from $\sigma^{*}\left(\mathrm{x}, \mathrm{q}^{C}\right)$ during the punishment phase if and only if

$$
\pi^{D P}(x)+\delta V^{P} \leq V^{P} .
$$

In other words, the one-shot deviation gain from the punishment phase plus the discounted payoff of staying in the punishment phase next period must not exceed the present value of abiding by the punishment rule (which guarantees a return to the collusive phase next period). Rearranging this inequality and using (4), we get the following incentive-compatibility constraint along the punishment path:

$$
\delta \geq \delta^{P}(x) \equiv \frac{\pi^{D P}(x)-\pi^{P}(x)}{\pi^{C}-\pi^{P}(x)} .
$$

The next lemma follows from the incentive-compatibility constraints.

LEMMA 1 The strategy profile $\sigma^{*}\left(\mathrm{x}, \mathrm{q}^{C}\right)$ is subgame-perfect if and only if

$$
\delta \geq \max \left\{\delta^{C}(x), \delta^{P}(x)\right\} .
$$

We are now ready to determine the set of punishment output levels $x$ for which the strategy profile $\sigma^{*}\left(\mathrm{x}, \mathrm{q}^{C}\right)$ forms a subgame-perfect equilibrium.

In the punishment phase, the outsider optimally responds to the punishment output $x$ and produces

$$
r_{0}(x)= \begin{cases}\frac{1-n x}{2} & \text { if } x<1 / n \\ 0 & \text { otherwise }\end{cases}
$$

Note that for $x \geq 1 / n$ the market price turns out to be nil, thereby driving the outsider out of business during the punishment period. Each firm within the cartel then obtains

$$
\pi^{P}(x)= \begin{cases}x\left[\frac{1-2 c-n x}{2}\right] & \text { if } x<1 / n \\ -c x & \text { otherwise. }\end{cases}
$$

Clearly, when $x \geq 1 / n$, the punishment payoff function $\pi^{P}(x)$ is negative because the market price is nil. When the market price is positive (i.e., when $x<1 / n), \pi^{P}(x)$ may 
still be negative due to dumping, with a market price that falls short of $c$. This happens when $x \geq(1-2 c) / n$. In addition, one can also observe that $\pi^{P}(x)$ is decreasing in $x$ for any $x \geq(1-2 c) / 2 n$. In any case, raising the punishment output floods the market, which exerts a downward pressure on the market price. Nevertheless, the loss entailed by the punishment should be recouped by reverting back to collusive behavior.

We can now determine the optimal deviation payoff for the firm that defects from the punishment phase. Given the outsider's best response to $x$, the payoff function for the deviator $i$ is given by

$$
\pi_{i}\left(r_{0}(x), x, \ldots, q_{i}, \ldots, x\right)= \begin{cases}\max \left\{0,\left[1-q_{i}-(n-1) x-\frac{1-n x}{2}-c\right] q_{i}\right\} & \text { if } x<1 / n, \\ \max \left\{0,\left[1-q_{i}-(n-1) x-c\right] q_{i}\right\} & \text { otherwise }\end{cases}
$$

Note that the firm that deviates from the punishment phase can always choose not to produce (with a payoff equal to 0 ) if it cannot get positive profits.

Maximizing the above payoff function with respect to $q_{i}$ yields the optimal deviation output during the punishment phase, i.e.,

$$
q^{D P}(x)= \begin{cases}\frac{1-2 c-x(n-2)}{4} & \text { if }\left\{\begin{array}{l}
\text { either } c<1 / n \text { and } x<1 / n, \\
\text { or } c \geq 1 / n \text { and } x<(1-2 c) /(n-2) \leq 1 / n,
\end{array}\right. \\
\frac{1-c-x(n-1)}{2} & \text { if } c<1 / n \text { and } 1 / n \leq x<(1-c) /(n-1), \\
0 & \text { otherwise. }\end{cases}
$$

When $x<1 / n$, the market price is positive and hence the outsider is better off producing $r_{0}(x)>0$, as one can see from (8). In this situation, the deviator also produces strictly positive quantities whenever $x<(1-2 c) /(n-2)$. When $c<1 / n$, we have $1 / n<$ $(1-2 c) /(n-2)$, so that $x<(1-2 c) /(n-2)$ whenever $x<1 / n$. If however $c \geq 1 / n$, we have $(1-2 c) /(n-2) \leq 1 / n$ and hence the relevant constraint is indeed given by $(1-2 c) /(n-2)$. When the punishment output is sufficiently large, viz. when $x \geq 1 / n$, the market price is equal to 0 and hence the best response of the outsider is to stay out of the market, i.e., $r_{0}(x)=0$. However, it might be possible that the behavior of the deviator, by producing a lower level of output than the agreed-on punishment output level, gives rise to a positive market price, so that the optimal deviation output is positive. For this situation to happen, one must have $x \in[1 / n,(1-c) /(n-1))$, which necessarily implies $1 / n<(1-c) /(n-1)$, i.e., $c<1 / n$. In all other cases, i.e., $c \geq 1 / n$ and $x \geq(1-2 c) /(n-2)$ or $c<1 / n$ and $x \geq(1-c) /(n-1)$, we have $q^{D P}(x)=0$.

Substituting (11) into (10) yields the optimal deviation payoff function

$$
\pi^{D P}(x)= \begin{cases}{\left[\frac{1-2 c-x(n-2)}{4}\right]^{2}} & \text { if }\left\{\begin{array}{l}
\text { either } c<1 / n \text { and } x<1 / n, \\
\text { or } c \geq 1 / n \text { and } x<(1-2 c) /(n-2) \leq 1 / n,
\end{array}\right. \\
{\left[\frac{1-c-x(n-1)}{2}\right]^{2}} & \text { if } c<1 / n \text { and } 1 / n \leq x<(1-c) /(n-1), \\
0 & \text { otherwise. }\end{cases}
$$


$\pi^{D P}(x)$ is always decreasing in $c$ whenever the market price induced by a deviation from the punishment path is positive. In other words, the larger the cost disadvantage of the cartel firms, the lower is the incentive to deviate from the punishment phase for any firm of the cartel even though the outsider is driven out of business, which happens when $c<1 / n$ and $1 / n \leq x<(1-c) /(n-1)$.

\section{The Most Collusive Symmetric Punishment (MCSP)}

We wish to characterize punishment output levels that maximize the scope for collusion among participating firms within the class of SSPEs. For this, we first determine the strongest credible punishment scheme, and then we restrict attention to the punishment output levels that supports collusion for the largest range of discount factors. We call that output level the most collusive symmetric punishment (MCSP).

First, as shown in the appendix (see Lemma A1 in appendix A.1), the punishment output level must be large enough and above a certain threshold - which will be given by $x_{d} \equiv 2(1-2 c) / 3 n$ - for the strategy profile $\sigma^{*}\left(\mathrm{x}, \mathrm{q}^{C}\right)$ to be an SSPE. Furthermore, the "optimal" output level should be greater than $q^{N}$ - which is itself greater than $x_{d}$ under Assumption 2 - otherwise the punishment would be lighter than Nash reversion. In the following, we will verify that this is indeed the case.

Thus, the strongest credible punishment scheme requires that $x \geq q^{N}$ be chosen to minimize the continuation value following a deviation $V^{P}$, subject to the incentive constraints (5) and (7). For this, the incentive constraint along the punishment phase must hold with equality, or equivalently $\delta=\delta^{P}(x)$. Suppose it does not. Then, the punishment output level $x$ can be raised so as to decrease both $\pi^{D P}(x)$ and $V^{P}$ until (6) holds with equality. ${ }^{6}$ Indeed, as $\pi^{P}(x)$ is decreasing in $x$ for any $x \geq(1-2 c) / 2 n$, we have that $V^{P}$ is also decreasing in $x$ for $x \geq q^{N}>x_{d}$, since $(1-2 c) / 2 n<x_{d}$. Furthermore, decreasing $V^{P}$ makes the incentive constraint along the collusive path, given by (5), more likely to be satisfied, because $\pi^{C}$ and $\pi^{D}$ do not depend on $x$.

Typically, there are multiple punishments such that both $\delta=\delta^{P}(x)$ and (5) hold. We focus on the punishment that supports collusion for the largest range of discount factors. This amounts here to characterizing the strongest credible punishment levels satisfying $\delta^{C}(x)=\delta^{P}(x)$, since $\pi^{P}(x)$ and thus $\delta^{C}(x)$ is decreasing in $x$. We then have the following lemma.

LEMMA 2 The most collusive symmetric punishment (MCSP), denoted by $\varkappa$, is the upper root of the equation $\delta^{C}(x)=\delta^{P}(x)$.

Neither payoff function $\pi^{P}(x)$ nor $\pi^{D P}(x)$ is everywhere differentiable and, moreover, $\pi^{D P}(x)$ is equal to zero for severe punishment output levels that drive the market price down to zero. Hence, the equation $\delta^{C}(x)=\delta^{P}(x)$ may admit several solutions. Therefore, we single out the highest value of $x$ satisfying this equality. This is the MCSP

${ }^{6}$ From (12), the payoff function $\pi^{D P}(x)$ is decreasing as $x$ increases, provided that the optimal deviation output is positive. 
denoted by $\varkappa$. Again, since $\pi^{P}(x)$ is decreasing in $x, \delta^{C}(x)$ is also always decreasing. As for $\delta^{P}(x)$, we cannot guarantee that it is always increasing. However, we will show that $\delta^{P}(x)$ is strictly increasing in $x$ on $[\varkappa,+\infty$ ) (see the proof of Proposition 1 ). Therefore, $\varkappa$ is the MCSP in that it most relaxes the two incentive compatibility constraints, thereby making it as easy as possible for cartel firms to collude.

First, using (5) and (7), the equation $\delta^{C}(x)=\delta^{P}(x)$ becomes $\pi^{D P}(x)-\pi^{P}(x)=$ $\pi^{D}-\pi^{C}$, which reduces (with (3)) to

$$
\pi^{D P}(x)-\pi^{P}(x)=\left[\frac{(1-2 c)(n-1)}{6 n}\right]^{2} .
$$

As a result, an MCSP requires equalizing the net gains of cheating in the collusive and the punishment phases. The symbols used for MCSPs in the remainder of the analysis are listed in Table 1.

Table 1

Notations for MCSPs

\begin{tabular}{cl}
\hline Variable & Definition \\
\hline$\varkappa_{1}^{s}$ & MCSP for which the deviator stays in the market in case 1 \\
$\varkappa_{1}^{e}$ & MCSP for which the deviator exits the market in case 1 \\
$\varkappa_{2}^{s}$ & MCSP for which the deviator stays in the market in case 2 \\
$\varkappa_{2}^{e}$ & MCSP for which the deviator exits the market in case 2 \\
\hline
\end{tabular}

Depending on the punishment output level, the market price can still be positive or nil. Therefore, we distinguish two cases. Case 1 corresponds to a situation where the market price is positive on the punishment path, which implies that the outsider produces positive quantities. There are two subcases depending on whether the firm that deviates from the punishment path exits the market - corresponding to the MCSP $\varkappa_{1}^{e}$ - or stays in the market - corresponding to the MCSP $\varkappa_{1}^{s}$. Case 2 corresponds to a situation where the market price (in the absence of any deviation) is nil on the punishment path, which implies that the outsider is driven out of business. Again, the firm that deviates from the punishment paths can exit the market - corresponding to the MCSP $\varkappa_{2}^{e}$ - or can stay in the market - corresponding to the MCSP $\varkappa_{2}^{s}$. In this last case, the lower production-level output than the agreed-on punishment output, due to the deviation, gives rise to a strictly positive market price (which would have been nil in the absence of a deviation).

We now introduce the functions in Table 2, which are useful for subsequent analysis. We then obtain the following proposition. ${ }^{7}$

\footnotetext{
7 The proof of Proposition 1 is given in Appendix A.2.
} 
Table 2

Limit Functions

\begin{tabular}{cl}
\hline Function & Definition \\
\hline $\mathrm{c}_{1}(n)$ & Lowest value of $c$ that satisfies $\varkappa_{2}^{e}-1 / n=0$, \\
& $\mathrm{c}_{1}(n)=\frac{n(n+7)+1-3 \sqrt{n(n+2)(2 n+1)}}{2(n-1)^{2}}$. \\
\hline $\mathrm{c}_{2}(n)$ & Lowest value of $c$ that satisfies $\varkappa_{2}^{e}-(1-c) /(n-1)=0$, \\
& $\mathrm{c}_{2}(n)=\frac{\sqrt{(n-1)^{3}+9 n^{2}}-3 n}{2 \sqrt{(n-1)^{3}+9 n^{2}}}$. \\
\hline $\mathrm{c}_{3}(n)$ & Highest value of $c$ that satisfies $\varkappa_{2}^{s}-1 / n=0$, \\
& $\mathrm{c}_{3}(n)=\frac{n-4}{5 n-2}$.
\end{tabular}

Proposition 1 The strategy profile $\sigma^{*}\left(\mathrm{x}, \mathrm{q}^{C}\right)$ admits a unique MCSP depending on the parameter configuration $(c, n)$, namely:

(i) if $n \geq 9$ : (ia) and $c \leq \mathrm{c}_{1}(n)$, then the MCSP is given by $\varkappa_{2}^{e}$; (ib) and $c>\mathrm{c}_{1}(n)$, then the MCSP is given by $\varkappa_{1}^{e}$, with $\varkappa_{1}^{e}<\varkappa_{2}^{e}$;

(ii) if $n \in\{7,8\}$ : (iia) and $c \leq \mathrm{c}_{2}(n)$, then the MCSP is given by $\varkappa_{2}^{e}$; (iib) and $c \in\left(\mathrm{c}_{2}(n), \mathrm{c}_{3}(n)\right]$, then the MCSP is given by $\varkappa_{2}^{s}$; (iic) and $c>\mathrm{c}_{3}(n)$, then the $M C S P$ is given by $\varkappa_{1}^{s}$, with $\varkappa_{1}^{s}<\varkappa_{2}^{s}<\varkappa_{2}^{e}$;

(iii) if $n \in\{5,6\}$ : (iiia) and $c \leq \mathrm{c}_{2}(n)$, then the MCSP is given by $\varkappa_{2}^{e}$; (iiib) and $c>\mathrm{c}_{2}(n)$, then the MCSP is given by $\varkappa_{1}^{s}$, with $\varkappa_{1}^{s}<\varkappa_{2}^{e}$;

where $\varkappa_{1}^{s}, \varkappa_{1}^{e}, \varkappa_{2}^{s}, \varkappa_{2}^{e}$ are given by

$$
\begin{aligned}
& \varkappa_{1}^{s}=\frac{(1-2 c)(5 n-2)}{3 n(n+2)}, \\
& \varkappa_{1}^{e}=\frac{(1-2 c)[3 n+\sqrt{n(n+2)(2 n+1)}]}{6 n^{2}}, \\
& \varkappa_{2}^{s}=\frac{3 n[(n-1)-c(n+1)]+\sqrt{(1-2 c)^{2}(n-1)^{4}-36 n^{2} c[(n-1)-c n]}}{3 n(n-1)^{2}}, \\
& \varkappa_{2}^{e}=\frac{1}{c}\left[\frac{(1-2 c)(n-1)}{6 n}\right]^{2} .
\end{aligned}
$$

Figure 1 maps out which MCSP emerges, depending on the size and the cost disadvantage of the cartel firms. As can be seen from this figure, there are four parameter regions in $(c, n)$ space, corresponding to four different punishment regimes, each giving rise to a specific MCSP.

First, in the most severe punishment regime, the MCSP $\varkappa_{2}^{e}$ drives the market price down to 0 , thereby driving the outsider out of business. In addition, the cartel firm that deviates from the punishment path cannot do better than cutting its production level 
Figure 1

The MCSPs

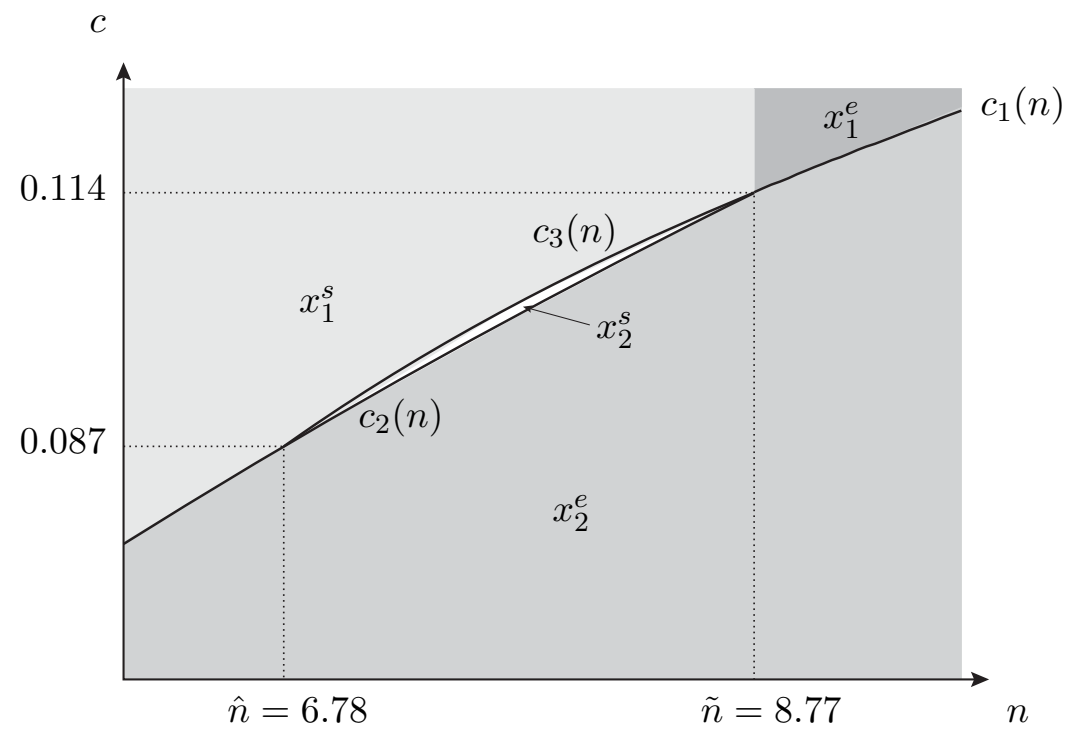

to 0 . Second, both punishment regimes corresponding to the MCSPs $\varkappa_{1}^{e}$ and $\varkappa_{2}^{s}$ are less severe than that corresponding to $\varkappa_{2}^{e}$. The MCSP $\varkappa_{1}^{e}$ induces the deviator to cut its production level to 0 , but the market price on the punishment path is positive, so that the outsider is better off remaining active in the market. As for $\varkappa_{2}^{e}$, the MCSP $\varkappa_{2}^{s}$ drives the market price down to 0 , but the deviator stays in the market and makes positive profits. The reason is that producing less than the punishment output level results in a positive market price. Third, the least severe punishment regime, corresponding to the MCSP $\varkappa_{1}^{s}$, allows both the outsider and the deviator to be active in the market. It is easily verified that $\varkappa_{1}^{s}$ is greater than $q^{N}$, so that all punishment schemes are more severe than Nash reversion.

Let us now investigate the comparative-static properties of the MCSPs with respect to $c$ and $n$. Changes in either of these exogenous parameters affect the level of the MCSP at work, and may also induce a switch from one punishment regime to another.

First consider changes in the cost disadvantage of cartel firms. One can easily check that the four MCSPs are decreasing in $c$. Moreover, Figure 1 shows that, when $n \geq 9$, the harshest MCSP is given by $\varkappa_{2}^{e}$ for $c \leq \mathrm{c}_{1}(n)$, while it is given by $\varkappa_{1}^{e}-$ with $\varkappa_{1}^{e}<\varkappa_{2}^{e}-$ for $c>\mathrm{c}_{1}(n)$. For $n<9$, the harshest MCSP is also given by $\varkappa_{2}^{e}$, when $c \leq \mathrm{c}_{2}(n)$. As the cost disadvantage increases above $\mathrm{c}_{2}(n)$, a lower punishment output level is sufficient to deter deviation from the collusive and punishment paths. Note that for $n \in\{7,8\}$, this lower punishment output level is given by $\varkappa_{2}^{s}$ for $c \in\left(c_{2}(n), c_{3}(n)\right]$, and $\varkappa_{1}^{s}$ for $c>c_{3}(n)$, while for $n \in\{5,6\}$, the MCSP is given by $\varkappa_{1}^{s}$ for any $c>\mathrm{c}_{2}(n) .{ }^{8}$ Intuitively, the larger the cost disadvantage of the cartel firms, the lower is the net gain of cheating in the collusive phase (i.e., $\pi^{D}-\pi^{C}$ ). Moreover, from (13), the punishment regime

${ }^{8}$ We show in the proof of Proposition 1 that $c_{3}(n)>c_{2}(n)$ for $n=\{7,8\}$ and that the reverse holds for $n<7$. This explains the statements of points (ii) and (iii) of this proposition. 
and the associated MCSP require that this net gain be equal to that of cheating in the punishment phase (i.e., $\pi^{D P}(x)-\pi^{P}(x)$ ). Thus, as $c$ increases, cartel firms resort to a less severe punishment regime in order to abide by the MCSP. We can summarize the discussion as follows.

COROLlary 1 For a given size of the cartel, the lower the cost disadvantage of the cartel firms, the more severe is the MCSP.

Unfortunately, the result is not so clear-cut when we look at the effect of the number of cartel firms on the levels of the MCSP for a given cost disadvantage of those firms. Clearly, $\varkappa_{2}^{e}$ - which holds for a sufficiently low cost disadvantage as shown in Figure 1 - is increasing in $n$. In contrast, $\varkappa_{1}^{e}$ and $\varkappa_{1}^{s}-$ which hold for sufficiently larger cost disadvantages - are both decreasing in $n$. Furthermore, computations show that the value of $\varkappa_{2}^{s}$ is lower for $n=8$ than for $n=7$. However, although we have monotonicity results of the MCSP with respect to the number of cartel firms within a specific punishment regime, we cannot be that conclusive when moving from one regime to another. We can only say that, if the cost disadvantage of the cartel is low enough, so that $\varkappa_{2}^{e}$ supports collusion (see Figure 1), then an increase in the number of cartel firms increases the punishment output level. In contrast, if the cost disadvantage of the cartel is sufficiently large, so that $\varkappa_{1}^{s}$ or $\varkappa_{1}^{e}$ supports collusion, then an increase in the size of the cartel within either punishment regime decreases the punishment output level. ${ }^{9}$ This shows the intricate effects of $n$ on the severity of the MCSP for a given level of the cost disadvantage of the cartel firms.

\section{The Minimum Discount Factor}

We can now determine the minimum discount factor such that the two incentive-compatibility constraints are binding. That is, we substitute the MCSP that solves (13) into (5) or (7). From the analysis of the previous section, we have that the MCSP can take four different expressions, depending on $c$ and $n$. First, consider the cases where the MCSP is given by $\varkappa_{1}^{e}$ or $\varkappa_{2}^{e}$. This corresponds to large punishment output levels where the cartel firm that deviates from the punishment path prefers not to produce when the price is positive or when it is nil, respectively. In either case, we have $\pi^{D P}\left(\varkappa_{2}^{e}\right)=\pi^{D P}\left(\varkappa_{1}^{e}\right)=0$. Then, from (13), we must have $-\pi^{P}\left(\varkappa_{2}^{e}\right)=-\pi^{P}\left(\varkappa_{1}^{e}\right)=\pi^{D}-\pi^{C}$. Using (5), we have that $\delta^{C}\left(\varkappa_{2}^{e}\right)=\delta^{C}\left(\varkappa_{1}^{e}\right)=\left[\pi^{D}-\pi^{C}\right] / \pi^{D}$. Using $\pi^{C}$ given by (1) and $\pi^{D}$ given by (2), we obtain

$$
\delta^{e}(n)=\frac{(n-1)^{2}}{(n+1)^{2}}
$$

Now, consider the case where the MCSP is given by $\varkappa_{1}^{s}$. This corresponds to a punishment output level where the cartel has less than nine firms and where the cartel firm

${ }^{9}$ Indeed, Figure 1 shows that the MCSP is $\varkappa_{1}^{s}$ when $c>\max \left\{\mathrm{c}_{2}(n), \mathrm{c}_{3}(n)\right\}$ and $n<9$; then, the MCSP becomes $\varkappa_{1}^{e}$ when $c>\mathrm{c}_{1}(n)$ and $n \geq 9$, with $\varkappa_{1}^{s}=\varkappa_{1}^{e}$ for $n=8.77$; and we know that $\varkappa_{1}^{s}$ and $\varkappa_{1}^{e}$ are both decreasing in $n$. 
that deviates from the punishment path produces positive quantities (but lower than those prescribed by the punishment level). We have $\pi^{P}\left(\varkappa_{1}^{s}\right)=\varkappa_{1}^{s}\left[\left(1-2 c-n \varkappa_{1}^{s}\right) / 2\right]$, where $\varkappa_{1}^{s}$ is given by (14). Substituting $\pi^{P}\left(\varkappa_{1}^{s}\right), \pi^{C}$, and $\pi^{D}$ into (5), we obtain

$$
\delta_{1}^{s}(n)=\frac{(n-1)(n+2)^{2}}{24 n(n-2)}
$$

which is lower than 1 for any $4 \leq n<9$.

Finally, consider the case where the MCSP is given by $\varkappa_{2}^{s}$. This corresponds to the situation where $n \in\{7,8\}$ and where the cartel firm that deviates from the punishment path also produces positive quantities. The difference with $\varkappa_{1}^{s}$ is that the firm that deviates from the punishment path - by producing a lower level of output than that prescribed by the punishment path - causes a positive market price that was at first nil. We then have $\pi^{P}\left(\varkappa_{2}^{s}\right)=-c \varkappa_{2}^{s}$, where $\varkappa_{2}^{s}$ is given by (14). Now, substituting $\pi^{P}\left(\varkappa_{2}^{s}\right)$, $\pi^{C}$, and $\pi^{C}$ into (5), we obtain

$$
\delta_{2}^{s}(n, c)=\frac{(1-2 c)^{2}(n-1)^{4}}{4 n\left[(1-2 c)^{2}-n[c(17 c+1)+2]+n^{2}[5 c(1-c)+1]+3 c \sqrt{(1-2 c)^{2}(n-1)^{4}-36 n^{2} c[(n-1)-c n]}\right]} .
$$

The next proposition summarizes these results.

Proposition 2 The minimal threshold for the discount factor above which the strategy profile $\sigma^{*}\left(\mathrm{x}, \mathrm{q}^{C}\right)$ forms an SSPE is given by:

(i) $\delta^{e}(n)$ if $n \geq 9$; or $n<9$ and $c \leq \mathrm{c}_{2}(n)$;

(ii) $\delta_{1}^{s}(n)$ if $n<9$ and $c>\max \left\{\mathrm{c}_{2}(n), \mathrm{c}_{3}(n)\right\}$;

(iii) $\delta_{2}^{s}(n, c)$ if $n \in\{7,8\}$ and $c \in\left(\mathrm{c}_{2}(n), \mathrm{c}_{3}(n)\right]$.

Figure 2 maps out which minimum discount factor emerges, depending on the size of the cartel and the cost disadvantage of the participating firms. We can now investigate the comparative-static properties of the minimum discount factor with respect to $c$ and $n$.

Keeping constant the size $n$ of the cartel, we begin by evaluating the impact of a change in $c$ on the difficulty of collusion within the cartel. First, observe that both $\delta^{e}(n)$ and $\delta_{1}^{s}(n)$ are independent of the cost disadvantage of the cartel. In other words, equalizing the net gains of cheating in the collusive and punishment phases may yield a punishment threat (i.e., the MCSP $\varkappa_{2}^{e}, \varkappa_{1}^{e}$, or $\varkappa_{1}^{s}$, depending on the parameter configuration), which makes the sustainability of the collusion within the cartel immune to its cost disadvantage. Yet, whether the relevant minimal discount factor is given by $\delta^{e}(n)$ or $\delta_{1}^{s}(n)$ depends on the relation between $c$ and $n$, as can be seen in Figure 2 . Furthermore, still depending on the parameter pair $(c, n)$, the minimum discount factor might also be given by $\delta_{2}^{s}(n, c)$, which is a function of $c$. Figure 2 shows that, if $n \in\{5,6\}$, the lowest discount factor is $\delta^{e}(n)$ when $c \leq c_{2}(n)$, but it is given by $\delta_{1}^{s}(n)$ when $c>\mathrm{c}_{2}(n)>\mathrm{c}_{3}(n) .{ }^{10}$ One can easily verify that $\delta_{1}^{s}(n)>\delta^{e}(n)$ for $n \in\{5,6\}$. If

\footnotetext{
${ }^{10}$ Again, we have that $\mathrm{c}_{3}(n)>\mathrm{c}_{2}(n)$ for $n=\{7,8\}$, while the reverse holds for $n<7$.
} 
Figure 2

Minimum Discount Factors

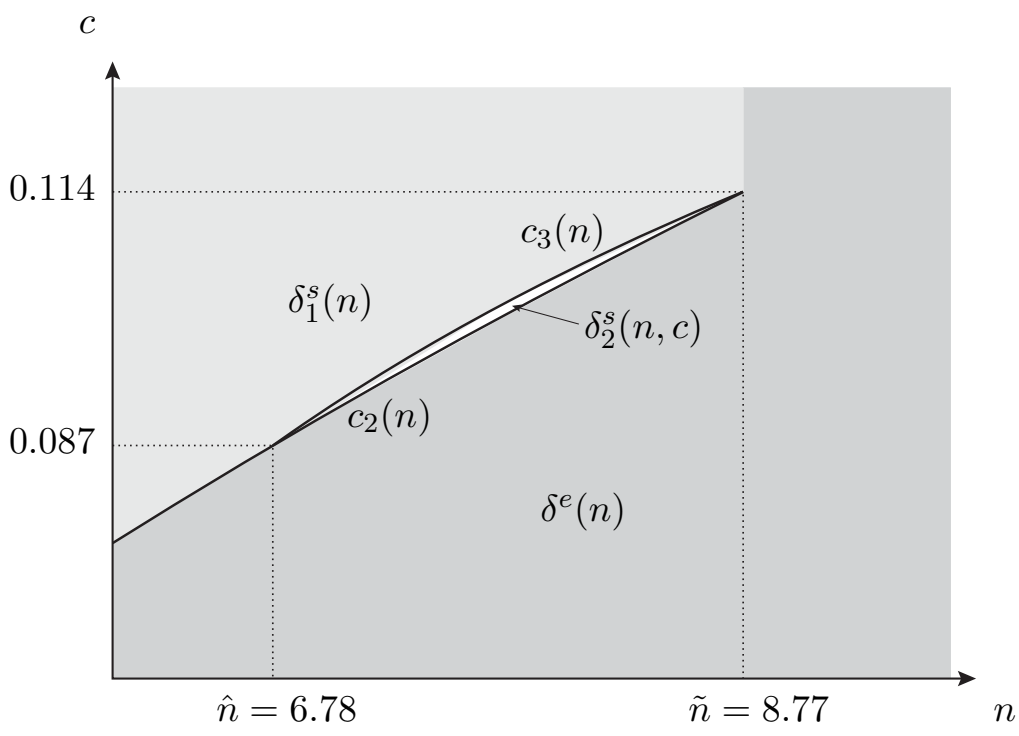

$n \in\{7,8\}$, then the lowest discount factor is $\delta^{e}(n)$ when $c \leq \mathrm{c}_{2}(n)$, whereas it is $\delta_{2}^{s}(n, c)$ when $c \in\left(\mathrm{c}_{2}(n), \mathrm{c}_{3}(n)\right]$, or $\delta_{1}^{s}(n)$ when $c>\mathrm{c}_{3}(n)>\mathrm{c}_{2}(n)$. We show in Appendix A.3 that $\delta_{2}^{s}(n, c)$ is increasing in $c$ and furthermore that $\delta^{e}(n)<\delta_{2}^{s}(n, c)<\delta_{1}^{s}(n)$ for $n \in\{7,8\}$. Finally, if $n \geq 9$, then the lowest discount factor is given by $\delta^{e}(n)$ independently of $c$. Therefore, collusion within the cartel is more difficult to sustain as the cost asymmetry increases, provided the size of the cartel is lower than nine firms. When the size of the cartel is larger than nine firms, then the cost disadvantage of the firms (relative to the outsider) has no effect on the difficulty in sustaining the collusion within the cartel. Yet, in this case, the MCSP depends on $c$, since it is given by $\varkappa_{2}^{e}$ for $c \leq \mathrm{c}_{1}(n)$ and by $\varkappa_{1}^{e}<\varkappa_{2}^{e}$ for $c>c_{1}(n)$. Corollary 2 summarizes the effects of changes in the cost disadvantage of the cartel on the difficulty of collusion.

COROLlary 2 When the cartel comprises less than nine firms, a lower cost disadvantage facilitates collusion. However, when the cartel comprises nine or more firms, the cost disadvantage has no effect on the difficulty of collusion.

The result that an increase in $c$ makes it more difficult to sustain collusion when the cartel includes less than nine firms is far from intuitive. Indeed, a decrease in the competitiveness of the cartel leads to a reduction in the net gain of cheating in the collusive phase (i.e., $\pi^{D}-\pi^{C}$ ), which would decrease the minimum discount factor. Nevertheless, from (5), we have that the minimum discount factor is inversely related to the net profit of collusion reflected by $\pi^{C}-\pi^{P}(x)$. This implies that, when $n<9$, the net profit of collusion decreases even more than the net gain of cheating in the collusive phase. Indeed, from Corollary 1, we know that a higher $c$ gives rise to different punishment regimes with less severe MCSPs that limit the scope for collusion and, finally, result in higher minimum discount factors. In contrast, when the cartel includes nine or 
more firms, switching to a less severe MCSP when $c$ increases entails no change in the minimum discount factor. This means that $\pi^{D}-\pi^{C}$ and $\pi^{C}-\pi^{P}(x)$ decrease at the same rate.

Now keeping constant the cost disadvantage $c$ of the cartel firms, one can also evaluate the impact of an increase in the size $n$ of the cartel. Figure 2 shows that, for $c \leq \mathrm{c}_{2}(n)$, the minimum discount factor is given by $\delta^{e}(n)$, which is always increasing in the size of the cartel (whether the cartel includes more or less than nine firms). Suppose now that $c>\mathrm{c}_{2}(n)$; then the minimum discount factor is given by $\delta_{1}^{s}(n)$ if $n \in\{5,6\}$, or if $n \in\{7,8\}$ and $c>\mathrm{c}_{3}(n)>\mathrm{c}_{2}(n)$, while it is given by $\delta_{2}^{s}(n, c)$ if $n \in\{7,8\}$ and $c \in\left(c_{2}(n), c_{3}(n)\right]$. For $c>c_{2}(n)$ and $n \geq 9$, the minimum discount factor is given by $\delta^{e}(n)$. We have that $\delta_{1}^{s}(n)$ is increasing in $n$ for $n \geq 5 .{ }^{11}$ Furthermore, we have that $\delta_{1}^{s}(8)=175 / 288<\delta^{e}(9)=16 / 25$, and we also show in the Appendix A.3 that $\delta_{1}^{s}(6)<\delta_{2}^{s}(7, c)<\delta_{2}^{s}(8, c)<\delta^{e}(9)$. Therefore, for any $c>\mathrm{c}_{2}(n)$, the difficulty of sustaining collusion is again increasing in the size of the cartel.

Figures 3 and 4 plot the critical discount factors as a function of $n$, given $c$. The curves are discontinuous because the MCSP is itself discontinuous. The difference between the two figures is that in Figure 3, for $n<9$, we can have $c \leq c_{2}(n)$ or $c>\max \left\{c_{2}(n), c_{3}(n)\right\}$. These two constraints on the value of $c$ (for $n<9$ ) exclude the restricted parameter configuration $n \in\{7,8\}$ and $c \in\left(\mathrm{c}_{2}(n), \mathrm{c}_{3}(n)\right]$. In contrast, in Figure 4, we consider that for $n \in\{7,8\}$, we have $c \in\left(\mathrm{c}_{2}(n), \mathrm{c}_{3}(n)\right]$ (all other values of $c$ being excluded for $n \in\{7,8\})$. This specific parameter configuration gives rise to the minimum discount factor $\delta_{2}^{s}(n, c)$. In both of these figures, we have also plotted $\delta^{m}(n)$ and $\delta^{m}(n+1)$. As we will see below, they give the minimum discount factors for sustaining an all-inclusive collusive agreement of, respectively, $n$ and $n+1$ identical firms in the class of stick-andcarrot equilibria. This will also allow us to illustrate some results about the secession of one firm from the collusive agreement (Corollary 4) or about the entry of a more competitive firm into the market (Corollary 5).

The next corollary summarizes the effects of changes in the cartel size on the difficulty of collusion within the cartel.

COROLlary 3 For a given cost disadvantage of the cartel firms, a reduction in the cartel size facilitates collusion.

One reason for this result is that a larger number of cartel firms strengthens the incentives to deviate from the collusive phase. Indeed, in a similar model - viz., in a Cournot market with linear demand and constant marginal costs, but with all firms being identical and participating in the collusive agreement - the critical discount factor is increasing in the number of firms involved in the agreement, whether firms use Nashreversion strategies (see Vives, 1999, p. 307) or stick-and-carrot strategies (see Motta, 2004, p. 171). A general explanation still available here is that a larger number of firms

11 The sign of the derivative of $\delta_{1}^{s}(n)$ with respect to $n$ is the same as the sign of $n^{3}-6 n^{2}+$ $6 n-4$. Using Mathematica, one obtains that it admits a unique real root given by $\bar{n} \simeq 4.95$, above which the polynomial is positive. 
Figure 3

The Minimum Discount Factors at the MCSP when: (i) $n \geq 9$ (in black), (ii) $n<9$ and $c \leq c_{2}(n)$ (in black), and (iii) $n<9$ and $c>\max \left\{c_{2}(n), c_{3}(n)\right\}$ (in gray)

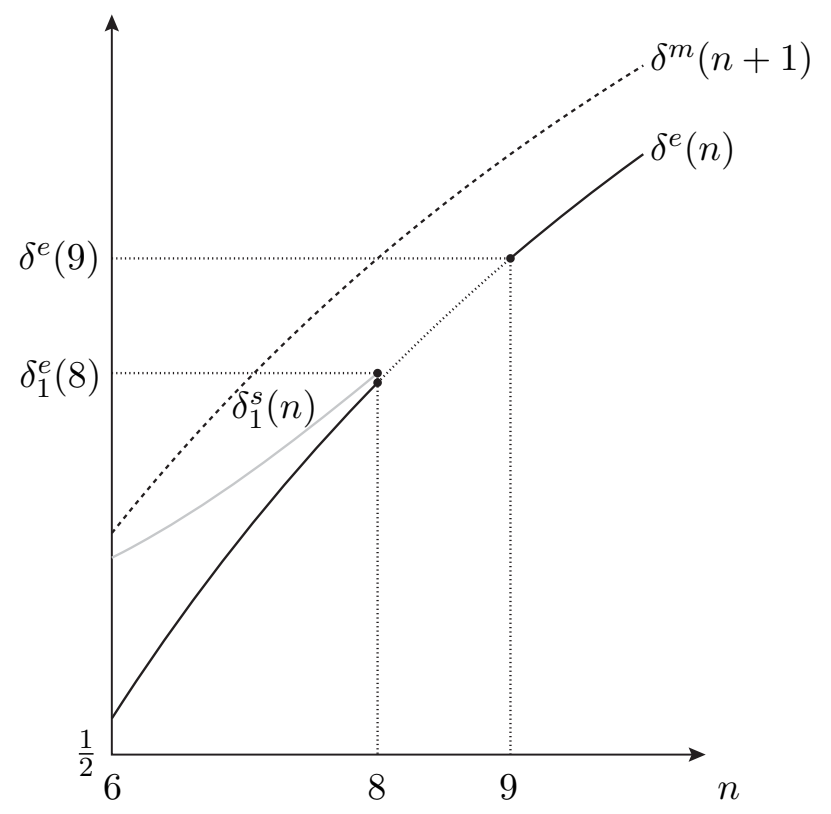

Figure 4

The Minimum Discount Factors at the MCSP when: (i) $n \geq 9$ (in black), (ii) $n<9$ and $c \leq c_{2}(n)$ (in black), and (iii) $c>c_{2}(n)$ if $n=6$ or $c \in\left(c_{2}(n), c_{3}(n)\right]$ and $n=\{7,8\}$ (dots in gray)

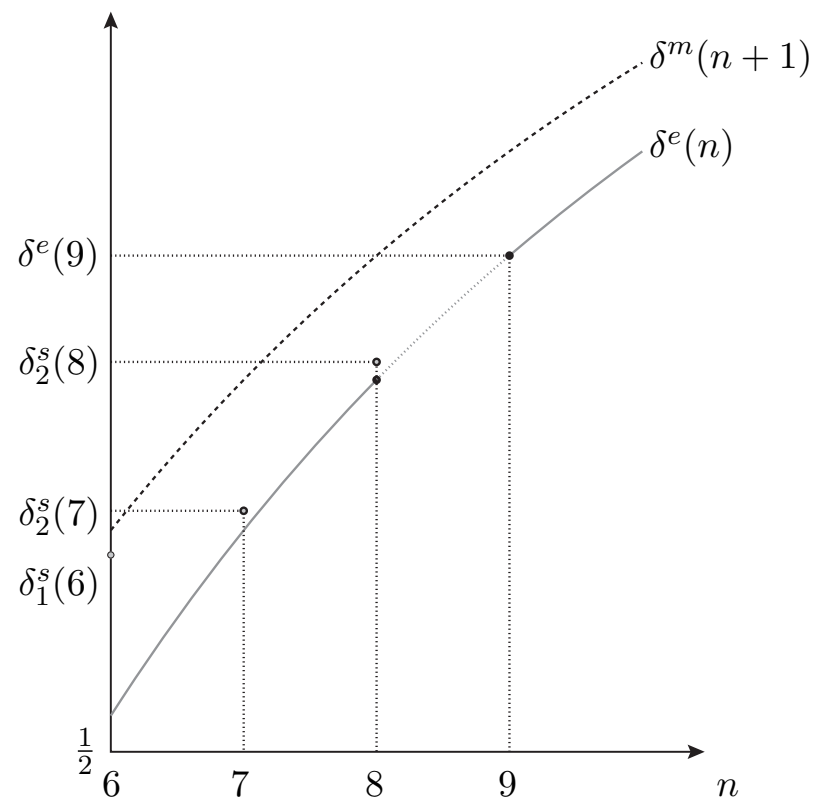


has the effect of increasing the net gain from deviating in the collusive phase, captured by $\pi^{D}-\pi^{C}$. One important difference with the traditional literature is that the deviating firm gains less in our setup, since the resulting outcome following the deviation is an (asymmetric) triopoly.

However, the explanation is not complete, because what matters is not only $\pi^{D}-\pi^{C}$ but the ratio $\delta^{C}(x)=\left(\pi^{D}-\pi^{C}\right) /\left(\pi^{C}-\pi^{P}(x)\right)$, which is equal to $\delta^{P}(x)$ at the MCSP. Hence, changes in $n$ also affect the net profit of collusion, which depends on the MCSP through $\pi^{P}(x)$. Recall that $\pi^{P}(x)$ is decreasing in $x$, and hence harsher punishments strengthen collusion. Nevertheless, we have previously seen that the MCSP can be either more or less severe when the cartel size increases. Hence, $\pi^{C}-\pi^{P}(x)$ can increase or decrease in $n$, depending on the punishment regime. In the case where this difference decreases - for example, within a punishment regime supported by $\varkappa_{1}^{s}$ or $\varkappa_{1}^{e}$, which are both decreasing in the cartel size - a greater number of cartel firms have weaker incentives to collude, which goes in the same direction as an increase of $\pi^{D}-\pi^{C}$ for raising the minimum discount factor. On the other hand, consider the most severe punishment regime supported by the MCSP $\varkappa_{2}^{e}$, which is increasing in $n$. As $\pi^{C}-\pi^{P}\left(\varkappa_{2}^{e}\right)$ also increases with the MCSP, the higher the number of cartel firms, the stronger the incentive to collude. Thus, the result that collusion is more difficult within a larger cartel implies that the increase in $\pi^{D}-\pi^{C}$ more than outweighs the increase in $\pi^{C}-\pi^{P}\left(\varkappa_{2}^{e}\right)$ resulting from a higher $n$.

Furthermore, it is interesting to compare the minimum discount factors with that sustaining collusion between $n+1$ identical firms in the class of stick-and-carrot equilibria, in the absence of an outsider. This comparison sheds light on a situation in which one cartel participant withdraws its collusion after obtaining a competitive advantage from an exogenous shock. With $y$ identical firms participating in the cartel agreement, the minimum discount factor is (see Motta, 2004, p. 171)

$$
\delta^{m}(y)= \begin{cases}\frac{(y+1)^{2}}{16 y} & \text { if } y<3+2 \sqrt{2}, \\ \frac{(y-1)^{2}}{(y+1)^{2}} & \text { otherwise. }\end{cases}
$$

First, observe that $\delta^{m}(n)$ is equal to $\delta^{e}(n)$, which holds (in our analysis) for any $n \geq 9$ independently of $c$, or for $n<9$ and $c \leq c_{2}(n)$. Now substituting $n+1$ for $y$ in (15), it can be seen in Figures 3 and 4 that $\delta^{m}(n+1)$ is higher than the different threshold values of the discount factor in the presence of an outsider, provided that $n \geq 6$ (thus $n+1 \geq 7$ ). We can thus establish the following corollary. ${ }^{12}$

Corollary 4 Suppose that the cartel includes at least seven firms. The secession of one firm from the collusive agreement - because of the occurrence of an (exogenous) cost

12 The proof of Corollary 4 is given in Appendix A.4. As shown in this proof, the statement does not hold for $n=5$ (thus $n+1=6$ ). It does not hold either for a cartel of less than five firms, since then the exit of one firm would lead to a cartel of (less than) four firms only. This is excluded by Assumption 2 because of the merger paradox. 
advantage - facilitates collusion among the remaining (identical) firms.

The mutation of a cartel firm into an advantaged outsider decreases the gain from collusion but also weakens the incentive to deviate from collusion as a result of the presence of an outside competitor. Corollary 4 shows that the latter effect dominates the former, making collusion against a more efficient outsider easier to sustain than all-inclusive collusion among (at least) six identical firms. Indeed, the presence of the outsider in case of a deviation is more painful to the deviator than would be the lack of competition in the absence of the outsider. The situation described is likely to be common in markets for primary commodities where the cohesion and strength of collusion between small producers is an issue (see, e.g., Gilbert, 1996). If one producer is endowed with a competitive advantage due to exogenous government subsidies, tacit collusion based on stick-and-carrot strategies may be a useful device to retaliate in the hands of rival producers - especially in some of the poorest regions of the world - which find themselves at a competitive disadvantage because they are not subsidized.

Finally, considering that $y=n$ in (15), we can also compare the minimum discount factor sustaining collusion among $n$ identical firms, with those obtained in our framework. This provides insight into a situation in which the cartel faces the entry of a more competitive firm in the market (but not in the cartel). It can be seen in Figures 3 and 4 that $\delta^{m}(n)$ never exceeds the different threshold values of the discount factor at the MCSP in the presence of an outsider, provided that $n \geq 6$. In particular, for $n \geq 9$ or $n<9$ and $c \leq c_{2}(n)$, the relevant discount factor is $\delta^{e}(n)$, which again is equal to $\delta^{m}(n)$. We thus have the following corollary. ${ }^{13}$

Corollary 5 Suppose that the cartel includes at least six firms. Collusion within the cartel is never easier to sustain after the entry of a more competitive firm into the market, independently of the size of the cartel.

The entry of the outsider decreases the gain from collusion among the $n$ cartel participants. It turns out that this effect can dominate the positive externality exerted by the outsider on the cartel collusion due to the lower attractiveness of deviation from the collusive phase. As a result, the entry of a more efficient outsider into the market does not facilitate collusion within the cartel.

\section{Conclusion}

In this paper, we have investigated the extent to which tacit collusion within a cartel of identical firms is affected by the presence of a more efficient outsider firm. The cartel is assumed to adopt two-phase punishment schemes in the style of Abreu $(1986,1988)$ to sustain the joint-profit-maximizing outcome, while the outsider is assumed to play its one-period best response to the cartel firms' strategies in every period. We focused on the maximal punishment regime that can be enforced, referred to as the MCSP, and

${ }^{13}$ The proof of Corollary 5 is given in Appendix A.5. As shown in that proof, the statement does not hold for $n=5$. 
then determined the minimal threshold value for the discount factor above which perfect collusion can be sustained as a (stationary) subgame-perfect equilibrium.

An important insight to be gained from this analysis is that the firms' ability to sustain collusion in the presence of a more competitive and noncooperative firm depends crucially, and in a quite complex way, on the number of firms involved in the agreement and on their cost disadvantage relative to the nonparticipating firm. Indeed, the MCSP and the corresponding minimal discount factor result from the interplay between the firms' incentives to deviate from the collusive agreement, on the one hand, and their incentives to deviate from the punishment phase, on the other. The overall insight is that the MCSP requires less severe punishments when the cost disadvantage of the cartel firm is relatively large. At lower levels of the MCSP, the minimum of patience needed to collude is higher than if firms were at a lower competitive disadvantage. Hence, collusion proves more difficult although deviation is less beneficial. By contrast, when the cartel is smaller, collusion is easier, essentially because deviation from the collusive phase is less beneficial. This positive effect on collusion more than offsets the negative effect due to the lax punishment. Finally, we provide insights into how the ease of collusion is affected either by the secession of a more efficient firm from the collusive agreement, or by the entry of a more efficient outsider into the market (but not into the cartel).

One interesting extension would be to assume that the outsider has a strategic behavior more sophisticated than just playing noncooperatively in each period of the game. For example, the outsider and a firm cheating on the cartel agreement might wish to collude. Alternatively, since collusion within the cartel benefits the outsider, one could imagine that the latter takes part in the punishment.

\section{Appendix}

\section{A.1 Lemma A1}

The following lemma states that the punishment output must be large enough for the strategy profile $\sigma^{*}\left(\mathrm{x}, \mathrm{q}^{C}\right)$ to be an SSPE.

LEMma A1 A necessary condition for the strategy profile $\sigma^{*}\left(\mathrm{x}, \mathrm{q}^{C}\right)$ to be an SSPE is that $x \geq x_{d}$, where $x_{d} \equiv 2(1-2 c) / 3 n$ is the highest solution to $\pi^{C}-\pi^{P}(x)=0$.

This lemma results from two underlying conditions. The first one is that we must have $\pi^{C}-\pi^{P}(x) \geq 0$ for (5) to be satisfied; otherwise each firm of the cartel would have an incentive to deviate from $q^{C}$ during the collusive phase so as to enter the punishment phase and get higher profits. Clearly, when $x>1 / n, \pi^{C}-\pi^{P}(x)$ is positive, since $\pi^{P}(x)=$ $-c x<0$. When $x \leq 1 / n$, we have $\pi^{C}-\pi^{P}(x)=(1 / 18 n)[3 n x-2(1-2 c)][3 n x-(1-2 c)]$. Hence, $\pi^{C}-\pi^{P}(x)=0$ has two solutions: $x_{d}^{\prime}=(1-2 c) / 3 n$ and $x_{d}=2(1-2 c) / 3 n$. Furthermore, the second derivative of $\pi^{C}-\pi^{P}(x)$ with respect to $x$ is positive, which implies that this function has a global minimum. It follows that $\pi^{C}-\pi^{P}(x)$ is positive if and only if $x \in\left[0, x_{d}^{\prime}\right] \cup\left[x_{d},+\infty\right)$. We now show that, for any $x \in\left[0, x_{d}^{\prime}\right]$, the incentivecompatibility constraint (7) in the punishment phase cannot be satisfied. Indeed, to 
ensure subgame perfection, the second condition is that $\pi^{D P}(x) \leq \pi^{C}$; otherwise the cheater would deviate in any period to get a greater profit than that obtained along the collusive path by triggering the same defection scenario forever. Since $x_{d}^{\prime}<1 / n$, we have $x<1 / n$ for any $x \in\left[0, x_{d}^{\prime}\right]$, and hence $\pi^{D P}(x)=[(1-2 c-x(n-2)) / 4]^{2}$. This function is decreasing on $[0,(1-2 c) /(n-2)]$, and then increasing for any $x \geq(1-2 c) /(n-2)$. Since $x_{d}^{\prime}<(1-2 c) /(n-2), \pi^{D P}(x)$ is decreasing on $\left[0, x_{d}^{\prime}\right]$ and reaches a minimum in $x_{d}^{\prime}$ on $\left[0, x_{d}^{\prime}\right]$. Evaluating at $x_{d}^{\prime}$ the profit of the deviator along the punishment path, one obtains $\pi^{D P}\left(x_{d}^{\prime}\right)=[(1-2 c)(n+1) / 6 n]^{2}$, which is greater than $\pi^{C}$. (The inequality $\pi^{D P}\left(x_{d}^{\prime}\right) \geq \pi^{C}$ reduces to $(n-1)^{2} \geq 0$.) It follows that the incentive-compatibility constraint (7) cannot be satisfied for $x \in\left[0, x_{d}^{\prime}\right]$. Therefore, a necessary condition for the two incentivecompatibility constraints to be simultaneously satisfied is that $x \in\left[x_{d},+\infty\right)$.

\section{A.2 Proof of Proposition 1}

To prove Proposition 1, we distinguish between two cases: case 1 where $c \geq 1 / n$, and case 2 where $c<1 / n$.

\section{A.2.1 Case $1(c \geq 1 / n)$ : A Relatively High Competitive Disadvantage of the Cartel}

$c \geq 1 / n$ is equivalent to $(1-2 c) /(n-2) \leq 1 / n$. In this situation, as shown by (12), the best deviation profit from the punishment path can be equal to 0 (for $x \geq(1-2 c) /(n-2)$ ) even though the market price is positive (for $x<1 / n$ ). When $c \geq 1 / n$, there are three types of punishment regimes, depending on the severity of the punishment output level relative to $(1-2 c) /(n-2)$ and $1 / n$. When the punishment output level is higher than $1 / n$, the market price falls to 0 and the outsider is driven out of business (see (8)), while the deviator also decides to cut its production level to 0 (see (11)). The MCSP that solves (13) in $[1 / n,+\infty)$ is denoted by $\varkappa_{2}^{e}$ whenever it exists. If not, we turn to less severe punishment levels inside $[(1-2 c) /(n-2), 1 / n)$. In this case, the market price is positive and the outsider is better off remaining active in the market. But again, in this case, the best deviation from the punishment path is to cut the production level to 0 . The MCSP that solves $(13)$ in $[(1-2 c) /(n-2), 1 / n)$ is denoted by $\varkappa_{1}^{e}$ whenever it exists. Otherwise, given Lemma A1, we focus on less severe subgame-perfect punishments within the interval $\left[x_{d},(1-2 c) /(n-2)\right)$, with $x_{d} \equiv 2(1-2 c) / 3 n$. In this case, the market price is still positive and both the outsider and the deviator are active in the market. The MCSP, if it exists, that solves $(13)$ in $\left[x_{d},(1-2 c) /(n-2)\right)$ is denoted by $\varkappa_{1}^{s}$. To summarize, we have $\varkappa_{1}^{s}<\varkappa_{1}^{e}<\varkappa_{2}^{e}$. We now establish the following lemma.

Lemma A2 Assume that $c \geq 1 / n$. Furthermore, let $\mathrm{c}_{1}(n)$ be the lowest value of $c$ that satisfies $\varkappa_{2}^{e}-1 / n=0$. Then, the strategy profile $\sigma^{*}\left(\mathrm{x}, \mathrm{q}^{C}\right)$ admits a unique MCSP given by: (i) $\varkappa_{2}^{e}$ if $n \geq 9$ and $c \leq \mathrm{c}_{1}(n)$; (ii) $\varkappa_{1}^{e}$ if $n \geq 9$ and $c>\mathrm{c}_{1}(n)$; (iii) $\varkappa_{1}^{s}$ if $n<9$.

Again, when $c \geq 1 / n$, we have $\pi^{D P}(x)=0$ for any $x \geq(1-2 c) /(n-2)$ as shown by (12). However, the payoff function $\pi^{P}(x)$ during the punishment phase depends on whether $x \in[(1-2 c) /(n-2), 1 / n)$ or $x \geq 1 / n$ as shown by (9). Suppose first that 
$x \geq 1 / n$, which implies $\pi^{P}(x)=-c x$. In that case, (13) has only one solution:

$$
\varkappa_{2}^{e}=\frac{1}{c}\left[\frac{(1-2 c)(n-1)}{6 n}\right]^{2} .
$$

The punishment output $\varkappa_{2}^{e}$ is relevant if $\varkappa_{2}^{e} \geq 1 / n$. The function $\varkappa_{2}^{e}-1 / n$ is quadratic in $c$, and therefore $\varkappa_{2}^{e}-1 / n=0$ has two roots, namely,

$$
\mathrm{c}_{1}(n)=\frac{n(n+7)+1-3 \sqrt{n(n+2)(2 n+1)}}{2(n-1)^{2}}
$$

and

$$
\mathrm{C}_{1}(n)=\frac{n(n+7)+1+3 \sqrt{n(n+2)(2 n+1)}}{2(n-1)^{2}} .
$$

Furthermore, the second derivative of $\varkappa_{2}^{e}-1 / n$ with respect to $c$ is positive, which implies that this function has a global minimum in $c$. Therefore, we have $\varkappa_{2}^{e} \geq 1 / n$ for any $c \notin\left(c_{1}(n), \mathrm{C}_{1}(n)\right)$. First, recall that Assumption 1 states that $c<1 / 2$, which guarantees that any firm has a positive market share in the stage game. Second, one can easily verify that $\mathrm{c}_{1}(n)<1 / 2$, this inequality being equivalent to $(n-1)^{2}>0$, and that $\mathrm{C}_{1}(n) \geq 1 / 2$. It follows that one can have $\varkappa_{2}^{e} \geq 1 / n$ only for $c \leq \mathrm{c}_{1}(n)$.

Since we are in the case $c \geq 1 / n$, one must also verify that $c_{1}(n) \geq 1 / n$. We have

$$
\mathrm{c}_{1}(n)-1 / n=\frac{n^{3}+5 n^{2}+5 n-2-3 n \sqrt{n(n+2)(2 n+1)}}{2 n(n-1)^{2}} .
$$

The numerator of this expression is positive if $\left[n^{3}+5 n^{2}+5 n-2\right]^{2} \geq 3 n^{3}(n+2)(2 n+1)$. This inequality can be equivalently rewritten as $(n-1)^{2}(n+2)(n+1)\left(n^{2}-9 n+2\right) \geq 0$, which is verified for any $n \geq \tilde{n}$ with $\tilde{n}=(9+\sqrt{73}) / 2 \simeq 8.77$.

Therefore, if $n \geq 9$ and $1 / n \leq c \leq \mathrm{c}_{1}(n)$, then $\varkappa_{2}^{e}$ (greater than $1 / n$ ) solves (13). Finally, to ensure that $\varkappa_{2}^{e}$ is the MCSP, we need to verify that $\delta^{P}(x)$ is increasing in $x$ for any $x \geq \varkappa_{2}^{e} \geq 1 / n$. Again, for any $x \geq(1-2 c) /(n-2)$, we have $\pi^{D P}(x)=0$, implying that $\delta^{P}(x)=-\pi^{P}(x) /\left[\pi^{C}-\pi^{P}(x)\right]$. Since $\pi^{P}(x)$ is always decreasing in $x$, clearly, $\delta^{P}(x)$ is increasing in $x$ whenever $x \geq(1-2 c) /(n-2)$. Since $\varkappa_{2}^{e} \geq 1 / n \geq(1-2 c) /(n-2)$, $\varkappa_{2}^{e}$ given by (A1) is indeed the MCSP when $n \geq 9$ and $1 / n \leq c \leq \mathrm{c}_{1}(n)$. This establishes point (i) of Lemma A2.

If $c>\mathrm{c}_{1}(n)$, then $\varkappa_{2}^{e}<1 / n$, implying that $\varkappa_{2}^{e}$ is not the MCSP. Then, if (13) admits a solution, it must be lower than $1 / n$. We now investigate whether there exists a solution within the interval $[(1-2 c) /(n-2), 1 / n)$. In that interval, we have $\pi^{P}(x)=$ $x[(1-2 c-n x) / 2]$ and still $\pi^{D P}(x)=0$. In this case, (13) has two roots. The lower root is $(1-2 c)[3 n-\sqrt{n(n+2)(2 n+1)}] / 6 n^{2}$, which is negative for any $n \geq 4$. The upper root is

$$
\varkappa_{1}^{e}=\frac{(1-2 c)[3 n+\sqrt{n(n+2)(2 n+1)}]}{6 n^{2}} .
$$

The punishment output $\varkappa_{1}^{e}$ is relevant only if $(1-2 c) /(n-2) \leq \varkappa_{e}<1 / n$. The inequality $\varkappa_{1}^{e}<1 / n$ reduces to $c>f(n)$, while the inequality $\varkappa_{1}^{e} \geq(1-2 c) /(n-2)$ reduces to 
$\left(n^{2}-9 n+2\right) \geq 0$, which is equivalent to the inequality $c_{1}(n) \geq 1 / n$ or $n \geq 9$. Therefore, if $n \geq 9$ and $c>c_{1}(n)>1 / n$, then $\varkappa_{1}^{e}$ solves $(13)$ on $[(1-2 c) /(n-2), 1 / n)$. Furthermore, this punishment level is the MCSP because, again, $\delta^{P}(x)$ is increasing in $x$ whenever $x \geq(1-2 c) /(n-2)$. This establishes point (ii) of Lemma A2.

Finally, suppose that $n<9$, implying that $c \geq 1 / n>c_{1}(n)$. In this case, neither $\varkappa_{2}^{e}$ nor $\varkappa_{1}^{e}$ can be the MCSP. If (13) admits a solution, then it must be lower than $(1-2 c) /(n-2)$, implying $\pi^{D P}(x) \geq 0$ and $V^{P} \geq 0$. In this case, we have $\pi^{P}(x)=$ $x[(1-2 c-n x) / 2]$ and $\pi^{D P}(x)=[(1-2 c-x(n-2)) / 4]^{2}$. Equation (13) has two roots, namely $(1-2 c) / 3 n$ and $(1-2 c)(5 n-2) / 3 n(n+2)$. The lower root does not satisfy Lemma A1, and hence the relevant solution is the upper root (satisfying Lemma A1), i.e.,

$$
\varkappa_{1}^{s}=\frac{(1-2 c)(5 n-2)}{3 n(n+2)} .
$$

One can check that $\varkappa_{1}^{s}<(1-2 c) /(n-2)$ reduces to $-n^{2}+9 n-2>0$, which implies that $n<9$. Finally, to ensure that $\varkappa_{1}^{s}$ is the MCSP, we need to verify that $\delta^{P}(x)=$ $\left[\pi^{D P}(x)-\pi^{P}(x)\right] /\left[\pi^{C}-\pi^{P}(x)\right]$ is also increasing in $x$ on $\left[\varkappa_{1}^{s},(1-2 c) /(n-2)\right)$. When $x<(1-2 c) /(n-2)$, we have

$$
\delta^{P}(x, n, c)=\frac{9 n[1-2 c-x(n+2)]^{2}}{8(1-2 c-3 n x)(2(1-2 c)-3 n x)} .
$$

Calculating the derivative of this expression, we have

$$
\frac{\partial \delta^{P}(x, n, c)}{\partial x}=\frac{9(1-2 c) n[1-2 c-x(n+2)][(1-2 c)(5 n-8)-9 n x(n-2)]}{8(1-2 c-3 n x)^{2}(2(1-2 c)-3 n x)^{2}} .
$$

This derivative is positive whenever $x \geq(1-2 c) /(n+2)>(1-2 c)(5 n-8) / 9 n(n-2)$. Since $\varkappa_{1}^{s}>(1-2 c) /(n+2)$, we have that $\delta^{P}(x)$ is increasing in $x$ on $\left[\varkappa_{1}^{s},(1-2 c) /(n-2)\right)$. This establishes point (iii) of Lemma A2.

\section{A.2.2 Case $2(c<1 / n)$ : A Relatively Low Competitive Disadvantage of the Cartel}

$c<1 / n$ is equivalent to $1 / n<(1-c) /(n-1)$. In this situation, as shown by (12), the best deviation profit from the punishment path can be positive (for $x<(1-c) /(n-1)$ ) even though the market price is equal to 0 in the absence of a deviation (for $x \geq 1 / n$ ). As in the previous case, there are three types of punishment regimes, depending on the severity of the punishment output level relative to $(1-c) /(n-1)$ and $1 / n$. First, when the punishment output level is higher than $(1-c) /(n-1)$, then both the market price and the best deviation profit from the punishment path are equal to 0. Furthermore, in this case, the profit of a firm that abides by the punishment rule is the same as that prevailing when $c \geq 1 / n$ and $x \geq 1 / n$. Therefore, the MCSP that solves (13) in $[(1-c) /(n-1),+\infty)$ must still be given by $\varkappa_{2}^{e}$, provided it exists. If not, we turn to less severe punishment output levels within the interval $[1 / n,(1-c) /(n-1))$. In this case, the market price is still equal to 0 if all firms of the cartel abide by the punishment rule. 
If however a firm decides to deviate from the punishment path, it produces a level of output lower than the agreed-on punishment level. This causes a positive market price, so that the deviator makes positive profits, as shown by (12). Provided it exists, the MCSP within the interval $[1 / n,(1-c) /(n-1))$ will be denoted by $\varkappa_{2}^{s}$. If it does not exist, then we look for a solution to (13) in the interval $\left[x_{d}, 1 / n\right)$, with $x_{d} \equiv 2(1-2 c) / 3 n$. In this case, the profit of a firm in the punishment phase and that of the deviator are identical to those obtained in the previous case, where $c \geq 1 / n$ and $\left[x_{d},(1-2 c) /(n-2)\right)$ (since now $1 / n<(1-2 c) /(n-2)$ ). Therefore, if it exists, the MCSP that solves (13) in $\left[x_{d}, 1 / n\right)$ must be given by $\varkappa_{1}^{s}$. To summarize, we will have $\varkappa_{1}^{s}<\varkappa_{2}^{s}<\varkappa_{2}^{e}$. We now establish the following lemma.

Lemma A3 Assume that $c<1 / n$. Furthermore, let $\mathrm{c}_{2}(n)$ be the lowest value of $c$ that satisfies $\varkappa_{2}^{e}-(1-c) /(n-1)=0$, and $\mathrm{c}_{3}(n)$ the highest value of $c$ that satisfies $\varkappa_{2}^{s}-1 / n=0$. Then, the strategy profile $\sigma^{*}\left(\mathrm{x}, \mathrm{q}^{C}\right)$ admits a unique MCSP given by (i) $\varkappa_{2}^{e}$ if $n \geq 9$ or if $n<9$ and $c \leq \mathrm{c}_{2}(n)$; (ii) $\varkappa_{2}^{s}$ if $n \in\{7,8\}$ and $c \in\left(\mathrm{c}_{2}(n), \mathrm{c}_{3}(n)\right]$; (iii) $\varkappa_{1}^{s}$ if $n \in\{7,8\}$ and $c>\mathrm{c}_{3}(n)>\mathrm{c}_{2}(n)$ or if $n \in\{5,6\}$ and $c>\mathrm{c}_{2}(n)>\mathrm{c}_{3}(n)$.

Again, when $c<1 / n$ and $x \geq(1-c) /(n-1)$, we have $\pi^{D P}(x)=0$ and $\pi^{P}(x)=-c x$, since $(1-c) /(n-1)>1 / n$. Again, (13) has one solution, given by (A1) . One must verify that $\varkappa_{2}^{e} \geq(1-c) /(n-1)$. The equation $\varkappa_{2}^{e}-(1-c) /(n-1)=0$ is quadratic in $c$ and hence has two roots, namely,

$$
c_{2}(n)=\frac{\sqrt{(n-1)^{3}+9 n^{2}}-3 n}{2 \sqrt{(n-1)^{3}+9 n^{2}}} \quad \text { and } \quad C_{2}(n)=\frac{\sqrt{(n-1)^{3}+9 n^{2}}+3 n}{2 \sqrt{(n-1)^{3}+9 n^{2}}} .
$$

Furthermore, the second derivative of $\varkappa_{2}^{e}-(1-c) /(n-1)$ with respect to $c$ is positive, which implies that this function has a global minimum in $c$. Therefore, we have $\varkappa_{2}^{e} \geq$ $(1-c) /(n-1)$ for any $c \notin\left(c_{2}(n), \mathrm{C}_{2}(n)\right)$. Again, one can observe that $\mathrm{C}_{2}(n)>1 / 2$, while $c_{2}(n)<1 / 2$. Since one must have $c<1 / 2$, one can have $\varkappa_{2}^{e} \geq(1-c) /(n-1)$ only for $c \leq \mathrm{c}_{2}(n)$.

Since we are in the case $c<1 / n$, we now evaluate the difference between $c_{2}(n)$ and $1 / n$. We have

$$
c_{2}(n)-1 / n=\frac{(n-2) \sqrt{(n-1)^{3}+9 n^{2}}-3 n^{2}}{2 n \sqrt{(n-1)^{3}+9 n^{2}}} .
$$

The numerator of this expression is positive if $(n-2)^{2}\left[(n-1)^{3}+9 n^{2}\right] \geq 9 n^{4}$. This inequality can be equivalently rewritten as $(n-1)^{2}(n+2)\left[n^{2}-9 n+2\right] \geq 0$, which is verified for any $n \geq \tilde{n}$, where $\tilde{n}=(9+\sqrt{73}) / 2 \simeq 8.77$. We then have $\mathrm{c}_{2}(n) \geq 1 / n$ for any $n \geq 9$, while the inequality is reversed for any $n<9$. Hence, when $c<1 / n$, $\varkappa_{2}^{e}$ solves (13) if $n \geq 9$ or if $n<9$ and $c \leq \mathrm{c}_{2}(n)<1 / n$. Finally, when $x \geq(1-c) /(n-1)$, we have $\delta^{P}(x)=-\pi^{P}(x) /\left[\pi^{C}-\pi^{P}(x)\right]$. Since $\pi^{P}(x)$ is always decreasing in $x, \delta^{P}(x)$ is increasing for any $x \geq(1-c) /(n-1)$. Therefore, when $c<1 / n$ and $n \geq 9$ or $n<9$ and $c \leq \mathrm{c}_{2}(n)<1 / n, \varkappa_{2}^{e}$ given by (A1) is the MCSP. This establishes point (i) of Lemma A3.

Suppose now that $n<9$ and $\mathrm{c}_{2}(n)<c<1 / n$, so that there does not exist a punishment output level higher than $\varkappa_{2}^{e}$ satisfying (13). We then look for a punishment 
level satisfying (13) lower than $(1-c) /(n-1)$. When $x<(1-c) /(n-1)$, the profit functions $\pi^{P}(x)$ and $\pi^{D P}(x)$ depend on whether $x \in[1 / n,(1-c) /(n-1))$ or $x<1 / n$, as shown by (9) and (12). Assume first that $x \in[1 / n,(1-c) /(n-1))$, implying $\pi^{P}(x)=-c x$ and $\pi^{D P}(x)=[(1-c-x(n-1)) / 2]^{2}$. Equation (13) then admits two roots, given by

$$
\varkappa_{2}^{s^{\prime}}=\frac{3 n[(n-1)-c(n+1)]-\sqrt{(1-2 c)^{2}(n-1)^{4}-36 n^{2} c[(n-1)-c n]}}{3 n(n-1)^{2}}
$$

and

$$
\varkappa_{2}^{s}=\frac{3 n(n-1)-c(n+1)]+\sqrt{(1-2 c)^{2}(n-1)^{4}-36 n^{2} c[(n-1)-c n]}}{3 n(n-1)^{2}} .
$$

We now show that $\varkappa_{2}^{s^{\prime}}$ given by (A5) does not satisfy Lemma A1, i.e., that $x_{d}^{\prime} \geq \varkappa_{2}^{s^{\prime}}$. The equation $x_{d}^{\prime}-\varkappa_{2}^{s^{\prime}}$ is quadratic in $c$ and has two roots, given by

$$
f(n)=\frac{\left(n^{2}+2 n-2\right)-n \sqrt{n^{2}+6 n+33}}{\left(n^{2}+16 n-4\right)}
$$

and

$$
F(n)=\frac{\left(n^{2}+2 n-2\right)+n \sqrt{n^{2}+6 n+33}}{\left(n^{2}+16 n-4\right)} .
$$

The lower root $f(n)$ is negative. Furthermore, the second derivative of $x_{d}^{\prime}-\varkappa_{2}^{s^{\prime}}$ with respect to $c$ is negative, which implies that this function has a global maximum in $c$. Therefore $x_{d}^{\prime}-\varkappa_{2}^{s^{\prime}}$ is positive (i.e., $x_{d}^{\prime} \geq \varkappa_{2}^{s^{\prime}}$ ) for any $c \in[0, F(n)]$. Since we are in the case $c<1 / n$, a sufficient condition to have $x_{d}^{\prime} \geq \varkappa_{2}^{s^{\prime}}$ is that $1 / n<F(n)$. A sufficient condition for this last inequality to be satisfied is that $1 / n<\left(n^{2}+2 n-2\right) /\left(n^{2}+16 n-4\right)$ or that $n[n(n+1)-18]+4>0$, which is indeed verified for any $n \geq 4$. It follows that $\varkappa_{2}^{s^{\prime}}$ does not satisfy Lemma A1.

The punishment output $\varkappa_{2}^{s}$ is relevant if $1 / n \leq \varkappa_{2}^{s}<(1-c) /(n-1)$. The inequality $\varkappa_{2}^{s}<(1-c) /(n-1)$ is equivalent to $c>\mathrm{c}_{2}(n)$. Furthermore, $\varkappa_{2}^{s}-1 / n$ is quadratic in $c$, and hence $\varkappa_{2}^{s}-1 / n=0$ has two roots, given by -1 and

$$
c_{3}(n)=\frac{n-4}{5 n-2} .
$$

Since the second derivative of $\varkappa_{2}^{s}-1 / n$ with respect to $c$ is negative, $\varkappa_{2}^{s}-1 / n$ has a global maximum and hence $\varkappa_{2}^{s} \geq 1 / n$ only for $c \leq \mathrm{c}_{3}(n) .{ }^{14}$

${ }^{14}$ With this constraint, we can now verify that the expression under the radical in the numerator of (A6), i.e., $(1-2 c)^{2}(n-1)^{4}-36 n^{2} c[(n-1)-c n]$, is positive for any $c \leq g(n)$. The derivative of this expression with respect to $c$ is $-4(1-2 c)^{2}(n-1)^{4}-36 n^{2}[(n-1)-2 c n]$, which is negative, since $c<1 / n<(n-1) / 2 n$ for any $n \geq 4$. Now replacing $c$ by $\mathrm{c}_{3}(n)$ in the expression under the radical in (A6), we obtain $\left[3\left(n^{3}-8 n^{2}+3 n-2\right) /(5 n-2)\right]^{2}$, which is strictly positive. This implies that the expression under the radical in the numerator of $(\mathrm{A} 6)$ is strictly positive for any $c \leq \mathrm{c}_{3}(n)$. 
Recall that we are now in the case $n<9$ and $\mathrm{c}_{2}(n)<c<1 / n$. First, one can observe that the sign of $c_{3}(n)-1 / n$ is the same as the sign of (again) $n^{2}-9 n+2$, which is negative for $n<9$ (thus implying $c_{3}(n)<1 / n$ ). Second, we must verify that $\mathrm{c}_{3}(n)>\mathrm{c}_{2}(n)$. We have

$$
c_{3}(n)-c_{2}(n)=\frac{3\left[n(5 n-2)-(n+2) \sqrt{(n-1)^{3}+9 n^{2}}\right]}{2(5 n-2)\left[\sqrt{(n-1)^{3}+9 n^{2}}\right]} .
$$

The numerator of this expression is positive if $-n^{5}+15 n^{4}-51 n^{3}-31 n^{2}-8 n+4 \geq 0$, or if $n^{3}\left(-n^{2}+15 n-51\right) \geq 31 n^{2}+8 n-4$. The right-hand member of this inequality is always positive, while the left-hand member is positive only if $n \in[(15-\sqrt{21}) / 2,(15+\sqrt{21}) / 2] \simeq$ $[5.21,9.79]$. Therefore, a necessary condition for $\mathrm{c}_{3}(n)-\mathrm{c}_{2}(n)$ to be positive is that $n=\{6,7,8\}$, since we have that $n<9$. However, one can easily verify that the above inequality is not satisfied for $n=6$, but only for $n=7$ or $n=8 .{ }^{15}$ Hence, $\varkappa_{2}^{s}$ solves (13) only for $n \in\{7,8\}$ and $\mathrm{c}_{2}(n)<c \leq \mathrm{c}_{3}(n)<1 / n$.

Finally, to ensure that $\varkappa_{2}^{s}$ is the MCSP, we need to verify that $\delta^{P}(x)=\left[\pi^{D P}(x)-\right.$ $\left.\pi^{P}(x)\right] /\left[\pi^{C}-\pi^{P}(x)\right]$ is also increasing in $x$ on $\left[\varkappa_{2}^{s},(1-c) /(n-1)\right)$. When $x<(1-$ $c) /(n-1)$, we have

$$
\delta^{P}(x, n, c)=\frac{9 n\left[4 c x+[(1-c)-x(n-1)]^{2}\right]}{4\left[9 n x c+(1-2 c)^{2}\right]} .
$$

Calculating the derivative of this expression with respect to $x$, we have

$$
\begin{aligned}
& \frac{\partial \delta^{P}(x, n, c)}{\partial x} \\
& \quad=\frac{9 n\left\{x(n-1)^{2}\left[9 n x c+2(1-2 c)^{2}\right]-(1+c)\left[c^{2}(n-8)-c(3 n-8)+2(n-1)\right]\right\}}{4\left[9 n x c+(1-2 c)^{2}\right]^{2}} .
\end{aligned}
$$

We need to show that the numerator of this expression is positive for any $x \geq \varkappa_{2}^{s}$. Denote by $\Psi(x, n, c)$ the function $\{\cdot\}$ in the numerator of this expression. Calculating the derivative of $\Psi(x, n, c)$ with respect to $x$, we obtain $\partial \Psi(x, n, c) / \partial x=18 n(n-1)^{2}[9 n x c+$ $\left.(1-2 c)^{2}\right]$, which is positive, implying that $\Psi(x, n, c)$ is increasing in $x$. Therefore, a sufficient condition for the numerator of $\partial \delta^{P}(x, n, c) / \partial x$ to be positive for any $x \geq \varkappa_{2}^{s}$ is that $\left[\Psi(x, n, c) \mid x=\varkappa_{2}^{s}\right] \geq 0$. Unfortunately, one cannot obtain the sign of $\Psi(x, n, c) \mid x=$ $\varkappa_{2}^{s}$ independently of $n$ and $c$. Therefore, we also calculate the derivative of $\Psi(x, n, c)$ with respect to $c$. We obtain

$$
\frac{\partial \Psi(x, n, c)}{\partial c}=x(n-1)^{2}[9 n x+8(1-2 c)]+(n-6)+24 c^{2}+n c(4-3 c),
$$

which is always positive, since $n \in\{7,8\}$ and $c<1 / n$. Therefore, a necessary and sufficient condition for $\partial \delta^{P}(x, n, c) / \partial x$ to be positive for any $x \geq \varkappa_{2}^{s}$ and $c \in\left(c_{2}(n), c_{3}(n)\right]$

${ }^{15}$ Using Mathematica, one finds that the relevant roots that solve $c_{3}(n)-c_{2}(n)=0$ are given by $\left.\hat{n}=[6+(378-3 \sqrt{1137}])^{1 / 3}+(378+3 \sqrt{1137})^{1 / 3}\right] / 3 \simeq 6.78$ and $\tilde{n}=(9+\sqrt{73}) / 2 \simeq 8.77$. 
is that $\left[\Psi(x, n, c) \mid x=\varkappa_{2}^{s}, c=\mathrm{c}_{2}(n)\right] \geq 0$ when $n=7$ and $n=8$. When $n=7$, we have

$$
\left.\Psi(x, n, c)\right|_{x=\varkappa_{2}^{s}, c=\mathrm{c}_{2}(n), n=7}=\frac{6174 \sqrt{73}(\sqrt{73}-7)}{5329} \simeq 15.28>0,
$$

while when $n=8$, we have

$$
\left.\Psi(x, n, c)\right|_{x=\varkappa_{2}^{s}, c=\mathrm{c}_{2}(n), n=8}=\frac{82944 \sqrt{919}(\sqrt{919}-24)}{844561} \simeq 18.80>0 .
$$

It follows that $\delta^{P}(x, n, c)$ given by (A8) is increasing in $x$ for $x \geq \varkappa_{2}^{s}$, and hence $\varkappa_{2}^{s}$ is the MCSP when $n \in\{7,8\}$ and $c \in\left(\mathrm{c}_{2}(n), \mathrm{c}_{3}(n)\right]$. This establishes point (ii) of Lemma A3.

Suppose now that $n \in\{7,8\}$ and $\mathrm{c}_{2}(n)<\mathrm{c}_{3}(n)<c<1 / n$. Then, neither $\varkappa_{2}^{e}$ nor $\varkappa_{2}^{s}$ can be the MCSP. If (13) admits a solution, then it must be lower than $1 / n$, implying $\pi^{D P}(x) \geq 0$ and $V^{P} \geq 0$. In this case, we have $\pi^{P}(x)=x[(1-2 c-n x) / 2]$ and $\pi^{D P}(x)=[(1-2 c-x(n-2)) / 4]^{2}$. As previously shown, (13) admits one solution satisfying Lemma A1, which is given by (A3) provided that $\varkappa_{1}^{s} \leq 1 / n$. One can easily check that this last constraint is equivalent to $c>c_{3}(n)$. Now, again assuming that $c>\mathrm{c}_{2}(n)$, suppose that $n<7$ (implying $1 / n>\mathrm{c}_{2}(n)>\mathrm{c}_{3}(n)$ ). In that case, we also have that neither $\varkappa_{2}^{e}$ nor $\varkappa_{2}^{s}$ can be the MCSP. Moreover, the inequality $c>c_{3}(n)$ is necessarily satisfied, since $c>\mathrm{c}_{2}(n)$. Thus, when $n \leq 7$ and $h(n)<c<1 / n$, the solution to (13) is also given by $\varkappa_{1}^{s}$. Finally, to ensure that $\varkappa_{1}^{s}$ is the MCSP, we need to verify that $\delta^{P}(x)=\left[\pi^{D P}(x)-\pi^{P}(x)\right] /\left[\pi^{C}-\pi^{P}(x)\right]$ is also increasing in $x$ on $\left[\varkappa_{1}^{s}, 1 / n\right)$. For any $x<1 / n, \delta^{P}(x)$ is still given by (A4), which has been shown to be increasing in $x$ for any $x \geq(1-2 c) /(n+2)$. Since $\varkappa_{1}^{s}>(1-2 c) /(n+2), \varkappa_{1}^{s}$ is the MCSP for $n \in\{7,8\}$ and $1 / n>c>c_{3}(n)>c_{2}(n)$ or $n \in\{5,6\}$ and $1 / n>c>c_{2}(n)>c_{3}(n)$. This establishes point (iii) of Lemma A3.

We now combine Lemma A2 and Lemma A3. Let first assume that $n \geq 9$. In this case, we have shown that $1 / n<\mathrm{c}_{1}(n)$ (see (A2)). Lemma A2 - point (i) - states that if in addition $1 / n \leq c \leq \mathrm{c}_{1}(n)$, then the MCSP is given by $\varkappa_{2}^{e}$. Lemma A3 - point (i) - states that for $c<1 / n$, the MCSP is also given by $\varkappa_{2}^{e}$. Since $c<1 / n$ implies that $c<\mathrm{c}_{1}(n)$, one can conclude that for $n \geq 9$ and $c \leq \mathrm{c}_{1}(n)$, the MCSP is given by $\varkappa_{2}^{e}$. If $c>\mathrm{c}_{1}(n)$, then that implies that $c>1 / n$. Lemma A2 - point (ii) - states that the MCSP is given by $\varkappa_{1}^{e}$. Therefore, when $n \geq 9$, the MCSP is given by $\varkappa_{2}^{e}$ for $c \leq \mathrm{c}_{1}(n)$, as stated in point (ia) of Proposition 1 , and by $\varkappa_{1}^{e}$ for $c>c_{1}(n)$, as stated in point (ib) of Proposition 1.

Assume now that $n \in\{7,8\}$. In this case, we have that $c_{1}(n)<1 / n$ (see (A2)). Lemma A2 - point (iii) - states that if $c \geq 1 / n$, then the MCSP is given by $\varkappa_{1}^{s}$. According to Lemma A3 - point (iii) - the MCSP is again given by $\varkappa_{1}^{s}$ for $1 / n>c>$ $\mathrm{c}_{3}(n)>\mathrm{c}_{2}(n)$. Therefore, for any $c>\mathrm{c}_{3}(n)$, the MCSP is given by $\varkappa_{1}^{s}$ as stated in point (iic) of Proposition 1. According to point (ii) of Lemma A3, the MCSP is given by $\varkappa_{2}^{s}$ for $c \in\left(\mathrm{c}_{2}(n), \mathrm{c}_{3}(n)\right]$, which corresponds to point (iib) of Proposition 1 . Now, if $c \leq \mathrm{c}_{2}(n)<1 / n$, Lemma A3 - point (i) - states that the MCSP is given by $\varkappa_{2}^{e}$, which corresponds to point (iia) of Proposition 1.

Finally, assume that $n \in\{5,6\}$. In this case, we still have that $c_{1}(n)<1 / n$ (see (A2)). Again, Lemma A2 - point (iii) - states that if $c \geq 1 / n$, then the MCSP is given 
by $\varkappa_{1}^{s}$. According to Lemma A3 - point (iii) - the MCSP is again given by $\varkappa_{1}^{s}$ for $1 / n>c>c_{2}(n)>c_{3}(n)$. Therefore, for any $c>c_{2}(n)$, the MCSP is given by $\varkappa_{1}^{s}$ as stated in point (iiib) of Proposition 1 . Now if $c \leq \mathrm{c}_{2}(n)<1 / n$, Lemma A3 - point (i) states that the MCSP is given by $\varkappa_{2}^{e}$, which corresponds to point (iiia) of Proposition 1 .

Q.E.D.

\section{A.3 The Minimum Discount Factor $\delta_{2}^{s}(n, c)$}

We show here that $\delta_{2}(n, c)$ is strictly lower than 1 and that it is increasing in $c$ for $c \in(h(n), g(n)]$ and $n=\{7,8\}$. When $n=7$, we have

$$
\delta_{2}^{s}(7, c)=\frac{18(1-2 c)^{2}}{7\left[\lambda_{1}(c)+c \sqrt{\lambda_{2}(c)}\right]},
$$

where $\lambda_{1}(c)=2+13 c-20 c^{2}$ and $\lambda_{2}(c)=36-438 c+487 c^{2}$. We have $\lambda_{1}^{\prime}(c)=13-40 c$, which is positive for any $c<1 / 7$. (Recall that $g(n)<1 / n$ for any $n<9$.) Hence, $\lambda_{1}(c)$ is increasing in $c$, so that a sufficient condition to have $\lambda_{1}(c)>0$ for any $c \in\left(\mathrm{c}_{2}(7), \mathrm{c}_{3}(7)\right]$ is that $\lambda_{1}(0)=2>0$. Similarly, we have $\lambda_{2}^{\prime}(c)=-438+974 c$, which is negative for any $n<1 / 7$. Hence, $\lambda_{2}(c)$ is decreasing in $c$ and reaches a minimum at $c=c_{3}(7)=1 / 11$. We have $\lambda_{2}(1 / 11)=25 / 121>0$, implying that $\lambda_{2}(c)$ is positive for any $c \in\left(c_{2}(7), c_{3}(7)\right]$. Furthermore, the derivative of $\delta_{2}^{s}(7, c)$ with respect to $c$ is given by

$$
\frac{\partial \delta_{2}^{s}(7, c)}{\partial c}=\frac{-18(1-2 c)\left[\lambda_{3}(c)+7(3-2 c) \sqrt{\lambda_{2}(c)}\right]}{7 \sqrt{\lambda_{2}(c)}\left[\lambda_{1}(c)+c \sqrt{\lambda_{2}(c)}\right]^{2}},
$$

where $\lambda_{3}(c)=36-585 c+536 c^{2}$. We have $\lambda_{3}^{\prime}(c)=-585+1072 c$, which is negative for any $c<1 / 7$. Hence, $\lambda_{3}(c)$ is decreasing in $c$, and furthermore is negative at $c=c_{2}(7)=$ $[\sqrt{73}-7] / 2 \sqrt{73}$, implying that $\lambda_{3}(c)$ is negative for any $c \in\left(c_{2}(7), c_{3}(7)\right]$. We then need to evaluate the sign of $\Psi(c)=\lambda_{3}(c)+7(3-2 c) \sqrt{\lambda_{2}(c)}$. We have $\Psi^{\prime}(c)<0$, since $\lambda_{2}^{\prime}(c)<0$ and $\lambda_{3}^{\prime}(c)<0$. Evaluating $\Psi(c)$ at $c=c_{2}(7)$, we have $\Psi(c)=0$, and hence $\Psi(c)$ is negative for any $c \in\left(\mathrm{c}_{2}(7), \mathrm{c}_{3}(7)\right]$. This implies that $\delta_{2}^{s}(7, c)$ is increasing in $c$ and that it reaches a maximum at $c=\mathrm{c}_{3}(7)=1 / 11$. We have $\left[\delta_{2}^{s}(7, c) \mid c=\mathrm{c}_{3}(7)\right]=729 / 1295 \simeq$ 0.5629 , and hence $\delta_{2}^{s}(7, c)<1$ for any $c \in\left(\mathrm{c}_{2}(7), \mathrm{c}_{3}(7)\right]$. Furthermore, $\delta_{2}^{s}(7, c)$ reaches a minimum at $c=c_{2}(7)$ and we have that $\left[\delta_{2}^{s}(7, c) \mid c=c_{2}(7)\right]=\delta^{e}(7)=9 / 16=0.5625$. Since we also have $\left[\delta_{2}^{s}(7, c) \mid c=c_{3}(7)\right]<\delta_{1}^{s}(7)=81 / 41 \simeq 0.5786$, we can conclude that $\delta^{e}(7)<\delta_{2}^{s}(7, c)<\delta_{1}^{s}(7)$ for any $c \in\left(\mathrm{c}_{2}(7), \mathrm{c}_{3}(7)\right]$.

When $n=8$, we have

$$
\delta_{2}^{s}(8, c)=\frac{2401(1-2 c)^{2}}{32\left[\lambda_{4}(c)+3 c \sqrt{\lambda_{5}(c)}\right]}
$$

where $\lambda_{4}(c)=49+308 c-452 c^{2}$ and $\lambda_{5}(c)=2401-25732 c+28036 c^{2}$. We have $\lambda_{4}^{\prime}(c)=308-904 c$, which is positive for any $c<1 / 8$. Hence, $\lambda_{4}(c)$ is increasing in $c$, so that a sufficient condition to have $\lambda_{4}(c)>0$ for any $c \in\left(c_{2}(8), c_{3}(8)\right]$ is that $\lambda_{4}(0)=49>0$. Similarly, we have $\lambda_{5}^{\prime}(c)=-25732+56072 c$, which is negative for any 
$n<1 / 7$. Hence, $\lambda_{5}(c)$ is decreasing in $c$, and reaches a minimum at $c=\mathrm{c}_{3}(8)=2 / 19$. We have $\lambda_{5}(2 / 19)=1089 / 361>0$, implying that $\lambda_{5}(c)$ is positive for any $c \in\left(c_{2}(8), c_{3}(8)\right]$. Furthermore, the derivative of $\delta_{2}^{s}(8, c)$ with respect to $c$ is given by

$$
\frac{\partial \delta_{2}^{s}(8, c)}{\partial c}=\frac{-7203(1-2 c)\left[\lambda_{6}(c)+24(7-4 c) \sqrt{\lambda_{5}(c)}\right]}{32 \sqrt{\lambda_{5}(c)}\left[\lambda_{4}(c)+3 c \sqrt{\lambda_{5}(c)}\right]^{2}}
$$

where $\lambda_{6}(c)=2401-33796 c+30340 c^{2}$. We have $\lambda_{6}^{\prime}(c)=-33796+60680 c$, which is negative for any $c<1 / 8$. Hence, $\lambda_{6}(c)$ is decreasing in $c$, and furthermore is negative at $c=h(8)=[\sqrt{919}-24] / 2 \sqrt{919}$, implying that $\lambda_{6}(c)$ is negative for any $c \in\left(c_{2}(8), c_{3}(8)\right]$. We then need to evaluate the sign of $\Lambda(c)=\lambda_{6}(c)+24(7-4 c) \sqrt{\lambda_{5}(c)}$. We have $\Lambda^{\prime}(c)<0$, since $\lambda_{5}^{\prime}(c)<0$ and $\lambda_{6}^{\prime}(c)<0$. Evaluating $\Lambda(c)$ at $c=\mathrm{c}_{2}(8)$, we have $\Lambda(c)=0$, and hence $\Lambda(c)$ is negative for any $c \in\left(c_{2}(8), c_{3}(8)\right]$. This implies that $\delta_{2}^{s}(8, c)$ is increasing in $c$ and that it reaches a maximum at $c=\mathrm{c}_{3}(8)=2 / 19$. We have $\left[\delta_{2}(8, c) \mid c=\mathrm{c}_{3}(8)\right]=175 / 288$, and hence $\delta_{2}(8, c)<1$ for any $c \in\left(c_{2}(8), c_{3}(8)\right]$. Furthermore, $\delta_{2}(8, c)$ reaches a minimum at $c=\mathrm{c}_{2}(8)$, and we have that $\left[\delta_{2}^{s}(8, c) \mid c=\mathrm{c}_{2}(8)\right]=\delta^{e}(8)=49 / 81 \simeq 0.6049$. Since we also have $\left[\delta_{2}^{s}(8, c) \mid c=\mathrm{c}_{3}(8)\right]=\delta_{1}^{s}(8)=175 / 188 \simeq 0.6076$, we can conclude that $\delta^{e}(8)<\delta_{2}^{s}(8, c)<\delta_{1}^{s}(8)$ for any $c \in\left(\mathrm{c}_{2}(8), \mathrm{c}_{3}(8)\right]$.

Finally, we compare $\delta_{2}^{s}(7, c), \delta_{2}^{s}(8, c), \delta_{1}^{s}(6)$ and $\delta^{e}(9)$. We have

$$
\delta_{2}^{s}(8, c)-\delta_{2}^{s}(7, c)=\frac{(1-2 c)\left[\lambda_{7}(c)+16807 c \sqrt{\lambda_{2}(c)}+1728 c \sqrt{\lambda_{5}(c)}\right]}{224\left[\lambda_{1}(c)+c \sqrt{\lambda_{2}(c)}\right]\left[\lambda_{4}(c)+3 c \sqrt{\lambda_{5}(c)}\right]},
$$

where $\lambda_{7}(c)=5390+41083 c-75788 c^{2}$. We have $\lambda_{7}^{\prime}(c)=41083-151576 c$, which is positive for any $c<1 / 7$. Hence, $\lambda_{7}(c)$ is increasing in $c$ and furthermore $\lambda_{7}(0)>0$. It follows that $\delta_{2}^{s}(8, c)-\delta_{2}^{s}(7, c)>0$ for any $c \in\left(\mathrm{c}_{2}(7), \mathrm{c}_{3}(7)\right] \subset\left(\mathrm{c}_{2}(8), \mathrm{c}_{3}(8)\right]$. Furthermore, the maximum of $\delta_{2}^{s}(8, c)$ is (again) $\left[\delta_{2}^{s}(8, c) \mid c=\mathrm{c}_{3}(8)\right]=175 / 288 \simeq 0.6076$, which is lower than $\delta^{e}(9)=(4 / 5)^{2}=0.64$. Finally, the minimum of $\delta_{2}^{s}(7, c)$ is (again) $\left[\delta_{2}^{s}(7, c) \mid c=\right.$ $\left.\mathrm{c}_{2}(7)\right]=9 / 16=0.5625$, which is greater than $\delta_{1}^{s}(6)=5 / 9 \simeq 0.5556$. We then have $\delta_{1}^{s}(6)<\delta_{2}^{s}(7, c)<\delta_{2}^{s}(8, c)<\delta^{e}(9)$.

Q.E.D.

\section{A.4 Proof of Corollary 4}

We compare the minimum discount factors of Proposition 2 - for sustaining collusion between $n$ firms with one advantaged firm outside the cartel - with the minimum discount factor $\delta^{m}(n+1)=n^{2} /(n+2)^{2}$ - for sustaining collusion between $n+1$ identical firms (without the outsider). First, we have $\delta^{m}(n+1)-\delta^{e}(n)=4\left(n^{2}+n-1\right) /\left(n^{2}+3 n+2\right)^{2}$, which is positive for any $n$, and where $\delta^{e}(n)$ holds for $n \geq 9$ or $n<9$ and $c \leq c_{2}(n)$. Suppose now that $n \in\{5,6\}$ and that $c>c_{2}(n)$, with $c_{2}(n)>c_{3}(n)$ for $n \in\{5,6\}$ (see (A7)); then the minimum discount factor with one breakaway firm is given by $\delta_{1}^{s}(n)$. We have $\delta^{m}(6)-\delta_{1}^{s}(5)=-151 / 4410<0$ but $\delta^{m}(7)-\delta_{1}^{s}(6)=1 / 144>0$. Now, suppose that $n \in\{7,8\}$. If furthermore $c>c_{3}(n)$, with $c_{3}(n)>c_{2}(n)$ for $n \in\{5,6\}$ (see (A7)), then the minimum discount factor with one breakaway firm is again given by $\delta_{1}^{s}(n)$. We have $\delta^{m}(8)-\delta_{1}^{s}(7)=299 / 11340$ and $\delta^{m}(9)-\delta_{1}^{s}(8)=71 / 2025$. If, however, $n \in\{7,8\}$ and 
$c \in\left(\mathrm{c}_{2}(n), \mathrm{c}_{3}(n)\right]$, then the minimum discount factor with one breakaway firm is given by $\delta_{2}^{s}(n, c)$. In the proof of Proposition 1, we show that $\delta_{2}^{s}(n, c)<\delta_{1}^{s}(n)$ for $n \in\{7,8\}$, which implies that $\delta^{m}(8)-\delta_{2}^{s}(7, c)$ and $\delta^{m}(9)-\delta_{2}^{s}(8, c)$ are (also) both positive. Therefore, for any $n \geq 6$ - i.e., a cartel of least seven firms - the breakaway of one firm makes collusion easier to sustain among the remaining firms.

Q.E.D.

\section{A.5 Proof of Corollary 5}

From (15), we have $\delta^{m}(n)=(n+1)^{2} / 16 n$ if $n=5$, i.e., $\delta^{m}(5)=9 / 20$, and $\delta^{m}(n)=$ $(n-1)^{2} /(n+1)^{2}$ for $n \geq 6$. If $c \leq c_{2}(n)$, then the minimum discount factor with one breakaway firm is given by $\delta^{e}(5)=4 / 9<\delta^{m}(5)$. If $c>c_{2}(n)$, then the corresponding minimum discount factor is given by $\delta_{1}^{s}(5)=49 / 90>\delta^{m}(5)$. Suppose now that $n \geq 9$ or that $n=6$ and $c \leq c_{2}(n)$; then relevant discount factor is given by $\delta^{e}(n)$, which is equal to $\delta^{m}(n)$. Now, if $n=6$ and $c>c_{2}(n)$ or $n \in\{7,8\}$ and $c>c_{3}(n)$, the relevant minimum discount factor is given by $\delta_{1}^{s}(n)$. We have

$$
\delta_{1}^{s}(n)-\delta^{m}(n)=\frac{(n-1)\left(n^{2}-9 n+2\right)^{2}}{24 n(n-2)(n+1)^{2}}>0 .
$$

Finally, if $n \in\{7,8\}$ and $c \in\left(c_{2}(n), c_{3}(n)\right]$, then the minimum discount factor with one breakaway firm is given by $\delta_{2}^{s}(n, c)$. In the proof of Proposition 1 , we show that $\delta_{2}^{s}(n, c)$ reaches a minimum at $c=c_{2}(n)$ and that $\left[\delta_{2}^{s}(n, c) \mid c=\mathrm{c}_{2}(n)\right]=\delta^{e}(n)=\delta^{m}(n)$ for $n \in\{7,8\}$. Therefore, for any $n \geq 6$, collusion within the cartel is never easier to sustain after the entry of a more competitive firm into the market (but not into the cartel).

Q.E.D.

\section{References}

Abreu, Dilip (1986), "Extremal Equilibria of Oligopolistic Supergames," Journal of Economic Theory, 39(1), 191-225.

- (1988), "On the Theory of Infinitely Repeated Games with Discounting," Econometrica, $56(2), 383-396$.

Baker, Jonathan B. (1995), "Fringe Firms and Incentives to Innovate," Antitrust Law Journal, $63(2), 621-641$.

Bos, Iwan, and Joseph E. Harrington, Jr. (2010), "Endogenous Cartel Formation with Heterogeneous Firms," The RAND Journal of Economics, 41(1), 92-117.

Escrihuela-Villar, Marc (2008a), "On Endogenous Cartel Size under Tacit Collusion," Investigaciones Económicas, 32(3), 325-338.

- (2008b), "Partial Coordination and Mergers among Quantity-Setting Firms," International Journal of Industrial Organization, 26(3), 803-810.

Friedman, James W. (1971), "A Non-Cooperative Equilibrium for Supergames," The Review of Economic Studies, 38(1), 1-12.

Gilbert, Christopher L. (1996), "International Commodity Agreements: An Obituary Notice," World Development, 24(1), 1-19. 
Griffin, J. M. (1989), "Previous Cartel Experience: Any Lessons for OPEC?" in: Lawrence R. Klein and Jaime Marquez (eds.), Economics in Theory and Practice: An Eclectic Approach, Kluwer Academic Publishers, Dordrecht, pp. 179-206.

Leibenstein, Harvey (1966), "Allocative Efficiency vs. 'X-Efficiency'," The American Economic Review, 56(3), 392-415.

Levenstein, Margaret C., and Valerie Y. Suslow (2006), "What Determines Cartel Success?" Journal of Economic Literature, 44(1), 43-95.

Miklós-Thal, Jeanine (2011), "Optimal Collusion under Cost Asymmetry," Economic Theory, 46(1), 99-125.

Motta, Massimo (2004), Competition Policy: Theory and Practice, Cambridge University Press, New York.

Rothschild, R. (1999), "Cartel Stability when Costs Are Heterogeneous," International Journal of Industrial Organization, 17(5), 717-734.

Salant, Stephen W., Sheldon Switzer, and Robert J.. Reynolds (1983), "Losses from Horizontal Merger: The Effects of an Exogenous Change in Industry Structure on Cournot-Nash Equilibrium," The Quarterly Journal of Economics, 98(2), 185-200.

Shaffer, Sherrill (1995), "Stable Cartels with a Cournot Fringe," Southern Economic Journal, $61(3), 744-754$.

Vasconcelos, Helder (2005), "Tacit Collusion, Cost Asymmetries, and Mergers," The RAND Journal of Economics, 36(1), 39-62.

Vives, Xavier (1999), Oligopoly Pricing: Old Ideas and New Tools, The MIT Press, Cambridge (MA).

Guillaume Cheikbossian

Faculté d'Economie

Université de Montpellier

(LAMETA) \& TSE

Avenue Raymond Dugrand - CS 79606

34960 Montpellier cedex 2

France

guillaume.cheikbossian@umontpellier.fr
Philippe Mahenc

Faculté d'Economie

Université de Montpellier

(LAMETA) \& TSE

Avenue Raymond Dugrand - CS 79606

34960 Montpellier cedex 2

France

philippe.mahenc@umontpellier.fr 